MATHEMATICS OF COMPUTATION

Volume 72, Number 241, Pages 55-81

S 0025-5718(02)01410-2

Article electronically published on May 1, 2002

\title{
AN ANALYSIS \\ OF NONCONFORMING MULTI-GRID METHODS, LEADING TO AN IMPROVED METHOD FOR THE MORLEY ELEMENT
}

\author{
ROB STEVENSON
}

\begin{abstract}
We recall and slightly refine the convergence theory for nonconforming multi-grid methods for symmetric positive definite problems developed by Bramble, Pasciak and $\mathrm{Xu}$. We derive new results to verify the regularity and approximation assumption, and the assumption on the smoother. From the analysis it will appear that most efficient multi-grid methods can be expected for fully regular problems, and for prolongations for which the energy norm of the iterated prolongations is uniformly bounded.

Guided by these observations, we develop a new multi-grid method for the biharmonic equation discretized with Morley finite elements, or equivalently, for the Stokes equations discretized with the $P_{0}$-nonconforming $P_{1}$ pair. Numerical results show that the new method is superior to standard ones.
\end{abstract}

\section{INTRODUCTION}

We reconsider the convergence theory for nonconforming multi-grid methods for symmetric positive definite problems developed by Bramble, Pasciak and $\mathrm{Xu}$ in [BPX91]. With nonconforming methods, the coarse-grid correction is not a projection, and in many applications it defines an iteration that is even divergent. As a consequence, a W-cycle multi-grid method is not a safe choice, since with a fixed number of smoothing steps it may result in a preconditioned system that is indefinite. On the other hand, a V-cycle type method yields preconditioned systems that are always positive definite. Moreover, for $m(k)$ denoting the number of preand post smoothing steps on level $k=1, \ldots, j$, it was proved that the resulting preconditioner is optimal when for some $\beta>1, m(k) \geq \beta^{j-k}$ (variable $\mathrm{V}$-cycle).

In this paper, we investigate to what extent this increase of the number of smoothing steps when going to coarser levels can be reduced, meanwhile preserving optimality. Apart from scientific interest, for a parallel implementation a reduction of the work on lower levels is important. A slight adaptation of the theory from BPX91] will show that for a "fully regular" problem, already $\sum_{k=0}^{j-1} \frac{1}{m(j-k)} \lesssim 1$ ensures optimality (mildly variable V-cycle) (cf. note at the end of this introduction).

Aiming at minimizing the work on lower levels, the best method is clearly the standard "nonvariable" V-cycle. Unfortunately, in the framework of nonconforming

Received by the editor November 23, 1998 and, in revised form, January 23, 2001.

2000 Mathematics Subject Classification. Primary 65N55, 65N30, 65F10.

Key words and phrases. Multi-grid method, nonconforming finite elements, biharmonic equation, Morley finite element space, Stokes equations. 
methods, we are not able to prove optimality of this cycle. Yet, we present an estimate that demonstrates that the condition number of the preconditioned system corresponding to the standard V-cycle may depend critically on the energy norm of the iterated prolongations alternated with smoothers. That is, if this norm grows exponentially with the numbers of levels, then the condition number may do so as well, whereas if this norm is uniformly bounded, then the standard Vcycle is at least suboptimal. These observations will be confirmed by numerical experiments. As a further support of these findings, we will recall some results obtained by Oswald in Osw97, which show that in order to get a suitable additive multi-grid method, it is essential to use prolongations for which the energy-norm of the iterated prolongations is uniformly bounded.

The main application that we will discuss is the biharmonic equation on some convex polygon, discretized with Morley elements. The biharmonic operator is not fully regular, which means that we can only rely on the variable V-cycle. Yet, as is well-known, the Stokes equations discretized with the $P_{0}$-nonconforming $P_{1}$ pair give rise to the same algebraic system. This equivalence has been exploited more often, in the sense that the biharmonic formulation was used to analyze multi-grid methods applied to the Stokes problem. Here we will follow the opposite approach.

The advantage of the Stokes formulation is that it defines a fully regular problem. Yet, since the usual basis for the finite element space is not uniformly $L^{2}$-stable, we have to pay for switching to this framework by the fact that standard smoothers do not satisfy the necessary assumptions. We develop a new type of smoothers that involve a call of a conforming multi-grid method to solve a discretized Laplacian. Using these smoothers, we show that the mildly variable V-cycle yields an optimal preconditioner.

It turns out that with the prolongation usually applied to the above-mentioned biharmonic, or equivalently Stokes problem, the energy norm of the iterated prolongations increases exponentially with the number of levels. We introduce a new prolongation, for which, at least in a model case, this energy-norm is uniformly bounded.

Using the standard V-cycle, we compare numerically the new smoother and prolongation with common choices. Both the new smoother and the new prolongation turn out to strongly reduce the condition numbers. With both improvements implemented, the condition numbers are "small", and they appear to be even uniformly bounded. Moreover, the new method can be implemented at the same costs as a standard method.

The remainder of this paper is organized as follows: We start by giving a description of the class of multi-grid methods that will be considered. This description is not only basis independent, but it also avoids making use of some scalar products, for which usually the $L^{2}$-scalar product is taken. As a consequence, the abstract formulation can be translated more easily in terms of an actual implementation.

We recall and slightly refine the multi-grid convergence theory from [BPX91. We give new, general applicable criteria to verify whether a smoother satisfies the assumption necessary for this convergence theory. The proofs are based on some simple algebraic arguments only. We give a short proof of a new theorem, requiring more or less minimal assumptions, to obtain the full regularity and approximation assumption. 
Mainly to underline the role of the iterated prolongations in the behaviour of multi-grid methods, we recall the convergence theory for additive multi-grid methods developed in Osw97. We relate the assumptions on the smoother in the multiplicative and the additive case.

Finally, we discuss some applications. Apart from the aforementioned application to the Morley element, we briefly discuss applications to the nonconforming $P_{1}$ and rotated $Q_{1}$ elements.

In order to avoid the repeated use of generic but unspecified constants, in this paper by $C \lesssim D$ we mean that $C$ can be bounded by a multiple of $D$, independently of parameters which $C$ and $D$ may depend on. Obviously, $C \gtrsim D$ is defined as $D \lesssim C$, and $C \equiv D$ as $C \lesssim D$ and $C \gtrsim D$.

Note. The referee pointed out that a publication of J.H. Bramble and X. Zhang is going to appear in which it is proved that, with $\alpha$ being the regularity parameter, optimality of a V-cycle type method is guaranteed when $\sum_{k=0}^{j-1} \frac{1}{m(j-k)^{\alpha}} \lesssim 1$, which generalizes our finding in the $\alpha=1$ case. Although this generalization means a quantitative improvement for $\alpha<1$, it is not in conflict with the approach followed in this paper to reformulate the less-regular biharmonic problem as a fully regular Stokes problem with the aim to reduce the number of necessary smoothing steps on lower levels.

\section{Multi-GRID MEthods}

2.1. Algorithm. We describe the symmetric (multiplicative) multi-grid method in a general setting. Let

$$
V_{0}, V_{1}, \ldots, V_{j}, \ldots,
$$

be a sequence of finite dimensional linear spaces over $\mathbb{R}$ or $\mathbb{C}$. By $V_{j}^{\prime}$ we denote the linear space of (anti-)linear functionals $g$ on $V_{j}$, i.e., $\bar{g}$ is linear. Assuming that $a_{j}$ is some scalar product on $V_{j}$, for given $g \in V_{j}^{\prime}$ we are interested in finding $u \in V_{j}$ such that

$$
a_{j}(u, v)=g(v) \quad\left(v \in V_{j}\right) .
$$

Defining $A_{j}: V_{j} \rightarrow V_{j}^{\prime}$ by

$$
\left(A_{j} u\right)(v)=a_{j}(u, v) \quad\left(u, v \in V_{j}\right)
$$

we see that (2.1) is equivalent to

$$
A_{j} u=g .
$$

To define a multi-grid method for solving (2.3) iteratively, for $1 \leq k \leq j$ we need suitable linear mappings $I_{k}: V_{k-1} \rightarrow V_{k}$, which are called prolongations. The dual mappings $I_{k}^{\prime}: V_{k}^{\prime} \rightarrow V_{k-1}^{\prime}$, defined by $\left(I_{k}^{\prime} g\right)(w)=g\left(I_{k} w\right)$, are then called restrictions.

Furthermore, to define the smoothers, for $1 \leq k \leq j$ we need possibly nonHermitian auxiliary sesquilinear forms $c_{k}$ on $V_{k}$, that give rise to operators $C_{k}, C_{k}^{\dagger}$ : $V_{k} \rightarrow V_{k}^{\prime}$ defined by

$$
\left(C_{k} u\right)(v)=c_{k}(u, v), \quad\left(C_{k}^{\dagger} u\right)(v)=\overline{c_{k}(v, u)} \quad\left(u, v \in V_{k}\right) .
$$

We set

$$
C_{k, \ell}= \begin{cases}C_{k} & \text { if } \ell \text { is odd } \\ C_{k}^{\dagger} & \text { if } \ell \text { is even }\end{cases}
$$


We assume that $\left\{u \in V_{k}: c_{k}\left(u, V_{k}\right)=0\right\}=\{0\}$, which means that $C_{k}$ and $C_{k}^{\dagger}$ are invertible.

The multi-grid operator $B_{k}: V_{k}^{\prime} \rightarrow V_{k}$ is now defined by induction as follows:

Let $B_{0}=A_{0}^{-1}$. Assume that $B_{k-1}$ has been defined and define $B_{k} g$ for $g \in V_{k}^{\prime}$ as follows:

1. Set $x^{(0)}=0$ and $q^{(0)}=0$.

2. Define $x^{(\ell)}$ for $\ell=1, \ldots, m(k)$ by

$$
x^{(\ell)}=x^{(\ell-1)}+C_{k, \ell+m(k)}^{-1}\left(g-A_{k} x^{(\ell-1)}\right) .
$$

3. Define $y^{(m(k))}=x^{(m(k))}+I_{k} q^{(p)}$, where $q^{(i)}$ for $i=1, \ldots, p$ is defined by

$$
q^{(i)}=q^{(i-1)}+B_{k-1}\left(I_{k}^{\prime}\left(g-A_{k} x^{(m(k))}\right)-A_{k-1} q^{(i-1)}\right) .
$$

4. Define $y^{(\ell)}$ for $\ell=m(k)+1, \ldots, 2 m(k)$ by

$$
y^{(\ell)}=y^{(\ell-1)}+C_{k, \ell+m(k)}^{-1}\left(g-A_{k} y^{(\ell-1)}\right) .
$$

5. Set $B_{k} g=y^{(2 m(k))}$.

Remark 2.1. The above description of the multi-grid method follows [BPX91] quite closely. A difference is that instead of using dual spaces $V_{k}^{\prime}$, in [BPX91] and in many other papers, after equipping the spaces $V_{k}$ with some additional scalar products $(,)_{0, k}$, all multi-grid components are defined between primal spaces by (implicitly) applying Riesz' representation theorem. Then, as a consequence, all these components depend on the particular choice of $(,)_{0, k}$, whereas the preconditioned system $B_{j} A_{j}$ does not. In other words, the influence of $(,)_{0, k}$ on the multi-grid algorithm is artificial. On the other hand, the introduction of suitable scalar products $(,)_{0, k}$ has turned out to be essential for the convergence theory.

A definition of the multi-grid algorithm directly in terms of its implementation, i.e., in terms of matrices and vectors, can for example be found in [Hac85]. Actually, when $(,)_{0, k}$ is the Euclidean scalar product corresponding to the basis one wants to apply, the definitions from [BPX91] and [Hac85] are similar.

Our definition is basis independent. Because it also does not depend on the scalar products $(,)_{0, k}$, the description of the implementation is straightforward, since it does not involve mass matrices and inverses of these matrices.

2.2. Implementation. For all $k$, let $V_{k}$ be equipped with some basis $\left\{\phi_{k, m}\right.$ : $\left.m \in J_{k}\right\}$, and think of $V_{k}^{\prime}$ as being equipped with the corresponding dual basis $\left\{\phi_{k, m}^{\prime}: m \in J_{k}\right\}$ defined by $\phi_{k, m}^{\prime}\left(\phi_{k, n}\right)=\delta_{n, m}$. From $g=\sum_{m \in J_{k}} g\left(\phi_{k, m}\right) \phi_{k, m}^{\prime}$, we see that $\mathbf{g}=\left(g\left(\phi_{k, m}\right)\right)_{m \in J_{k}}$ is the vector representation of $g \in V_{k}^{\prime}$. It is easily verified that the operator $A_{k}: V_{k} \rightarrow V_{k}^{\prime}$ is represented by the stiffness matrix $\mathbf{A}_{k}=\left(a\left(\phi_{k, n}, \phi_{k, m}\right)\right)_{m, n \in J_{k}}$, and that if $\mathbf{p}_{k}$ denotes the matrix representation of $I_{k}: V_{k-1} \rightarrow V_{k}$, then the matrix transpose $\mathbf{p}_{k}^{T}$ is the representation of $I_{k}^{\prime}$.

Noting that $a_{k}(u, v)=\left\langle\mathbf{A}_{k} \mathbf{u}, \mathbf{v}\right\rangle$, where the vectors $\mathbf{u}$ and $\mathbf{v}$ denote the representations of $u \in V_{k}$ and $v \in V_{k}$, respectively, and $\langle$,$\rangle is the Euclidean scalar product,$ a natural way to construct the sesquilinear forms $c_{k}$ is the following: For $\mathbf{C}_{k}$ being some (easily) invertible approximation of $\mathbf{A}_{k}$, define

$$
c_{k}(u, v)=\left\langle\mathbf{C}_{k} \mathbf{u}, \mathbf{v}\right\rangle \text {. }
$$

Then the representations of the operators $C_{k}, C_{k}^{\dagger}: V_{k} \rightarrow V_{k}^{\prime}$ are given by $\mathbf{C}_{k}$ and its matrix adjoint $\mathbf{C}_{k}^{H}$, respectively. 
2.3. Convergence theory. We recall convergence results obtained in BPX91], and give some additional estimates.

We will denote by ()$^{*}$ an adjoint with respect to the "energy" scalar product $a_{k}$. Then setting $K_{k}=I-C_{k}^{-1} A_{k}$, we have $K_{k}^{*}=I-C_{k}^{-\dagger} A_{k}$. Defining

$$
\tilde{K}_{k}^{(m)}= \begin{cases}\left(K_{k}^{*} K_{k}\right)^{m / 2} & \text { if } m \text { is even, } \\ \left(K_{k}^{*} K_{k}\right)^{(m-1) / 2} K_{k}^{*} & \text { if } m \text { is odd, }\end{cases}
$$

and noting that

$$
I_{k}^{*}=A_{k-1}^{-1} I_{k}^{\prime} A_{k}
$$

for $k>0$ we have

$$
I-B_{k} A_{k}=\left(\tilde{K}_{k}^{(m(k))}\right)^{*}\left[\left(I-I_{k} I_{k}^{*}\right)+I_{k}\left(I-B_{k-1} A_{k-1}\right)^{p} I_{k}^{*}\right] \tilde{K}_{k}^{(m(k))} .
$$

Note that $\left(B_{k} A_{k}\right)^{*}=B_{k} A_{k}$, and that $\left(\tilde{K}_{k}^{(m(k))}\right)^{*}\left(I-I_{k} I_{k}^{*}\right) \tilde{K}_{k}^{(m(k))}$ is the error amplification operator of the corresponding two-grid method.

For $(,)_{0, k}$ some additional scalar product on $V_{k}$, and $\|\cdot\|_{0, k}:=(\cdot, \cdot)_{0, k}^{\frac{1}{2}}$, we define

$$
\|u\|_{2, k}=\sup _{0 \neq v \in V_{k}} \frac{\left|a_{k}(u, v)\right|}{\|v\|_{0, k}} \quad\left(u \in V_{k}\right)
$$

and

$$
\rho_{k}=\sup _{0 \neq u \in V_{k}} \frac{a_{k}(u, u)}{\|u\|_{0, k}^{2}} .
$$

We make the following assumptions:

Regularity and Approximation Assumption. There exists an $\alpha \in(0,1]$ such that

$$
\left|a_{k}\left(\left(I-I_{k} I_{k}^{*}\right) u, u\right)\right| \lesssim\left(\rho_{k}^{-1}\|u\|_{2, k}^{2}\right)^{\alpha} a_{k}(u, u)^{1-\alpha} \quad\left(u \in V_{k}\right),
$$

and furthermore

$$
a_{k}(u, u)-a_{k}\left(K_{k} u, K_{k} u\right) \gtrsim \rho_{k}^{-1}\|u\|_{2, k}^{2} \quad\left(u \in V_{k}\right) .
$$

If in addition

$$
\rho\left(I_{k}^{*} I_{k}\right) \leq 1
$$

then the

- W-cycle, i.e., $p=2$, and $m(1)=\cdots=m(j) \geq 1$,

- variable V-cycle, i.e., $p=1$, and for some $\beta>1, m(j-k) \geq \beta^{k} m(j)$ and $m(j) \geq 1$,

and when $\alpha=1$, the

- standard V-cycle, i.e., $p=1$, and $m(1)=\cdots=m(j) \geq 1$,

all have been shown to yield $B_{j}$ that satisfy

$$
\sigma\left(I-B_{j} A_{j}\right) \subset\left[0, \frac{\delta_{j}}{1+\delta_{j}}\right], \text { where } \delta_{j} \lesssim m(j)^{-\alpha} .
$$

A particular case for which (C1) is valid is $I_{k}^{*} I_{k}=I$, i.e., $a_{k-1}(u, v)=a_{k}\left(I_{k} u, I_{k} v\right)$ ("Galerkin approach"). For this case a lot of additional multi-grid convergence theory is available, even some for which a regularity assumption is not necessary. In Che99] an application is described of the Galerkin approach to nonconforming finite element discretizations, which involves a redefinition of the energy scalar products on lower levels. 
If only

$$
\rho\left(I_{k}^{*} I_{k}\right) \leq 2
$$

then the W-cycle has been shown to yield $B_{j}$ that satisfy

$$
\sigma\left(I-B_{j} A_{j}\right) \subset\left[\frac{-\delta_{j}}{1+\delta_{j}}, \frac{\delta_{j}}{1+\delta_{j}}\right], \text { where } \delta_{j} \lesssim m(j)^{-\alpha} .
$$

Unfortunately, for the usual nonconforming multi-grid methods, (C1) does not hold, whereas (C2) has been shown only for an $I_{k}$ used for the rotated $Q_{1}$ element (CO98]). The common $I_{k}$ used for the nonconforming $P_{1}$ element or the Morley element generally do not satisfy (C2), and neither does the alternative $I_{k}$ for the Morley element that will be introduced in this paper.

Without (C2), the W-cycle satisfies (2.10) for $m(k)=m$ sufficiently large. In fact, it can be shown that it is sufficient that

$$
\rho\left(I_{k}^{*} \tilde{K}_{k}^{(m-1)}\left(\tilde{K}_{k}^{(m-1)}\right)^{*} I_{k}\right) \leq 2,
$$

which generalizes $(\mathrm{C} 2)$. By $(\mathrm{A})$ and $(\mathrm{B})$, the forthcoming Lemma 2.2 and $(2.13)$ show that indeed (C3) is valid for $m$ sufficiently large.

If (C3) is not valid, then the W-cycle may result in a preconditioned system that is indefinite. On the other hand, when $p=1$, from $(\mathrm{B})$ it can be deduced that $B_{j} A_{j}$ is positive definite anyway, although not necessarily $\rho\left(I-B_{j} A_{j}\right)<1$. In the remainder of this subsection, we will study some $V$-cycle variants, i.e., $p=1$, to be used as preconditioners, only assuming (A) and (B).

For the variable $\mathrm{V}$-cycle, i.e., $m(j-k)$ grows exponentially with $k$, it has been shown that

$$
\lambda_{\max }\left(I-B_{j} A_{j}\right) \leq \frac{\delta_{j}}{1+\delta_{j}}, \text { where } \delta_{j} \lesssim m(j)^{-\alpha} .
$$

Yet, from the analysis presented in [BPX91], it can be deduced that when $\alpha=1$, (2.11) is even valid for any $(m(k))_{1 \leq k \leq j}$ with $m(k) \geq m(j)$.

We now consider $\lambda_{\min }\left(I-B_{j} A_{j}\right)=-\lambda_{\max }\left(B_{j} A_{j}-I\right)$. A repeated use of (2.6) shows that

$$
a_{j}\left(\left(B_{j} A_{j}-I\right) u, u\right)=\sum_{k=1}^{j} a_{k}\left(\left(\tilde{K}_{k}^{(m(k))}\right)^{*}\left(I_{k} I_{k}^{*}-I\right) \tilde{K}_{k}^{(m(k))} \tilde{I}_{j \leftarrow k}^{*} u, \tilde{I}_{j \leftarrow k}^{*} u\right),
$$

where

$$
\tilde{I}_{j \leftarrow k}:=\left(\tilde{K}_{j}^{(m(j))}\right)^{*} I_{j} \tilde{I}_{j-1 \leftarrow k} \quad(j>k), \quad \text { and } \quad \tilde{I}_{k \leftarrow k}:=I .
$$

With

$$
q_{k}:=\max \left\{0, \lambda_{\max }\left(\left(\tilde{K}_{k}^{(m(k))}\right)^{*}\left(I_{k} I_{k}^{*}-I\right) \tilde{K}_{k}^{(m(k))}\right)\right\}
$$

we find that

$$
\lambda_{\max }\left(B_{j} A_{j}-I\right) \leq \sum_{k=1}^{j} q_{k} \rho\left(\tilde{I}_{j \leftarrow k}^{*} \tilde{I}_{j \leftarrow k}\right) .
$$

For convenience, from BPX91] we recall the following result that concerns the two-grid method:

Lemma 2.2. Assume that (A) and (B) hold. Then

$$
q_{k} \leq \rho\left(\left(\tilde{K}_{k}^{(m(k))}\right)^{*}\left(I-I_{k} I_{k}^{*}\right) \tilde{K}_{k}^{(m(k))}\right) \lesssim m(k)^{-\alpha} .
$$


Proof. With $\bar{K}_{k}=\left\{\begin{array}{ll}K_{k}^{*} K_{k} & \text { if } m(k) \text { is even } \\ K_{k} K_{k}^{*} & \text { if } m(k) \text { is odd }\end{array}\right.$, and (B) show that

$$
\begin{aligned}
\left|a_{k}\left(\left(I-I_{k} I_{k}^{*}\right) \tilde{K}_{k}^{(m(k))} u, \tilde{K}_{k}^{(m(k))} u\right)\right| & \lesssim\left(\rho_{k}^{-1}\left\|\tilde{K}_{k}^{(m(k))} u\right\|_{2, k}^{2}\right)^{\alpha} a_{k}\left(\bar{K}_{k}^{m(k)} u, u\right)^{1-\alpha} \\
& \lesssim a_{k}\left(\left(I-\bar{K}_{k}\right) \bar{K}_{k}^{m(k)} u, u\right)^{\alpha} a_{k}\left(\bar{K}_{k}^{m(k)} u, u\right)^{1-\alpha} .
\end{aligned}
$$

Since $\sigma\left(\bar{K}_{k}\right) \subset[0,1]$, which follows from $(\underline{B})$, we infer that

$$
a_{k}\left(\left(I-\bar{K}_{k}\right) \bar{K}_{k}^{m(k)} u, u\right) \leq a_{k}(u, u) \max _{\lambda \in[0,1]}(1-\lambda) \lambda^{m(k)} \lesssim m(k)^{-1} a_{k}(u, u)
$$

and $a_{k}\left(\bar{K}_{k}^{m(k)} u, u\right) \leq a_{k}(u, u)$, which completes the proof.

To estimate $\rho\left(\tilde{I}_{j \leftarrow k}^{*} \tilde{I}_{j \leftarrow k}\right)$, we use the fact that

$$
\begin{aligned}
& a_{k}\left(I_{k+1}^{*} \tilde{K}_{k+1}^{(m(k+1))} u, I_{k+1}^{*} \tilde{K}_{k+1}^{(m(k+1))} u\right) \\
& \quad=a_{k+1}\left(\left(\tilde{K}_{k+1}^{(m+1))}\right)^{*}\left(I_{k+1} I_{k+1}^{*}-I\right) \tilde{K}_{k+1}^{(m(k+1))} u, u\right)+a_{k+1}\left(\bar{K}_{k+1}^{m(k+1)} u, u\right) \\
& \quad \leq\left(q_{k+1}+1\right) a_{k+1}(u, u),
\end{aligned}
$$

and so $\rho\left(\tilde{I}_{j \leftarrow k}^{*} \tilde{I}_{j \leftarrow k}\right) \leq \prod_{i=k+1}^{j}\left(q_{i}+1\right)$. From (2.12) and Lemma 2.2, we conclude that

$$
\begin{aligned}
\lambda_{\max }\left(B_{j} A_{j}-I\right) & \leq \sum_{k=1}^{j} q_{k} \prod_{i=k+1}^{j}\left(q_{i}+1\right)=-1+\prod_{k=1}^{j}\left(q_{k}+1\right) \\
& \leq-1+e^{\sum_{k=1}^{j} q_{k}} \leq-1+e^{\sum_{k=0}^{j-1} m(j-k)^{-\alpha}} .
\end{aligned}
$$

For the variable $\mathrm{V}$-cycle, we infer that $\lambda_{\max }\left(B_{j} A_{j}-I\right) \lesssim m(j)^{-\alpha}$, and so by (2.11), $\kappa\left(B_{j} A_{j}\right)-1 \lesssim m(j)^{-\alpha}$, as was also noted in BPX91. However, a milder increase of $m(j-k)$ as a function of $k$ is already sufficient. For example, $m(j-k)$ $\gtrsim m(j)+k^{\beta}$ for some $\beta>\frac{1}{\alpha}$ yields $\lambda_{\max }\left(B_{j} A_{j}-I\right) \lesssim m(j)^{-\alpha+\frac{1}{\beta}}$. What is more, to get $\lambda_{\max }\left(B_{j} A_{j}-I\right)$ to be uniformly bounded, it is obviously already sufficient that $\sum_{k=0}^{j-1} m(j-k)^{-\alpha} \lesssim 1$. Combining this with the bound (2.11) on $\lambda_{\max }\left(I-B_{j} A_{j}\right)$, which for $\alpha=1$ is valid for any $(m(k))_{1 \leq k \leq j}$ with $m(k) \geq m(j)$, yields the following result:

Theorem 2.3 ("mildly" variable V-cycle). Assume (A) with $\alpha=1$ and (B). Let $p=1, m(k) \geq m(j)$, and $\sum_{k=0}^{j-1} \frac{1}{m(j-k)} \lesssim 1$. Then $\kappa\left(B_{j} A_{j}\right) \lesssim 1$.

So, besides the fact that upper bounds on the condition number corresponding to various cycles decrease faster as functions of the number of smoothing steps when $\alpha$ is larger, Theorem 2.3 is another indication that for more regular problems one may expect more efficient multi-grid methods, in particular when $\alpha=1$.

Aiming at minimizing the number of operations on lower levels, obviously the best algorithm is the standard $V$-cycle. Unfortunately, a proof of optimality of this cycle applied to general nonconforming discretizations cannot be deduced from the above estimates. However, some useful observations can be made. As stated before, for $\alpha=1$ the upper bound (2.11) on $\lambda_{\max }\left(I-B_{j} A_{j}\right)$ is also valid for the standard V-cycle. Furthermore, again for the standard V-cycle, (2.12) shows that the behaviour of $\lambda_{\max }\left(B_{j} A_{j}-I\right)$ might depend critically on the factors $\rho\left(\tilde{I}_{j \leftarrow k}^{*} \tilde{I}_{j \leftarrow k}\right)$, 
that is, on the squared energy norm of the "iterated prolongations alternated with smoothers". Indeed, in case these factors are uniformly bounded, then at least $\lambda_{\max }\left(B_{j} A_{j}-I\right) \lesssim j m(j)^{-\alpha}$, and so $\kappa\left(B_{j} A_{j}\right) \lesssim j m(j)^{-1}$ if $\alpha=1$. However, if $\rho\left(\tilde{I}_{j \leftarrow k}^{*} \tilde{I}_{j \leftarrow k}\right)$ increases exponentially with $j-k$ (which is not excluded by the preceding analysis), then $\kappa\left(B_{j} A_{j}\right)$ might increase exponentially as a function of $j$.

Numerical results for an $\alpha=1$ case presented in Section 3.3.3 show that this exponential increase of the condition number indeed occurs with a prolongation that is commonly used. With a new prolongation, that is developed with the aim of getting bounded factors $\rho\left(\tilde{I}_{j \leftarrow k}^{*} \tilde{I}_{j \leftarrow k}\right)$, the condition number of the standard $\mathrm{V}$-cycle even turns out to be uniformly bounded.

2.4. Assumption (B) for inexact Gauss-Seidel and damped Jacobi smoothers. By substituting $u=A_{k}^{-1} C_{k} w$, we see that (B) can be rewritten as

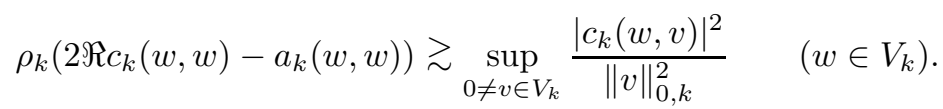

Having fixed some bases of the spaces $V_{k}$, and with $c_{k}$ and $\mathbf{C}_{k}$ related according to (2.5), and mass matrices $\mathbf{M}_{k}$ defined by $\left\langle\mathbf{M}_{k} \mathbf{u}, \mathbf{v}\right\rangle=(u, v)_{0, k}\left(u, v \in V_{k}\right)$, by noting that

$$
\rho_{k}=\rho\left(\mathbf{M}_{k}^{-1} \mathbf{A}_{k}\right)
$$

(2.14) in turn can be written as

$$
\rho\left(\mathbf{M}_{k}^{-1} \mathbf{A}_{k}\right)\left(\mathbf{C}_{k}+\mathbf{C}_{k}^{H}-\mathbf{A}_{k}\right) \gtrsim \mathbf{C}_{k}^{H} \mathbf{M}_{k}^{-1} \mathbf{C}_{k} .
$$

In two propositions, we give sufficient conditions for $(\mathrm{B})$. Dealing with "inexact smoothers", i.e., smoothers that involve an inexact, possibly nonsymmetric inner solver, these propositions generalize results from the literature, e.g., from BP92. These generalizations turn out to be particularly useful in cases where the mass matrices are not uniformly well-conditioned, a situation that we will encounter in practical applications.

Proposition 2.4. Let $\tilde{\mathbf{D}}_{k}, \mathbf{D}_{k}$ and $\mathbf{L}_{k}$ be some matrices of the same size as $\mathbf{A}_{k}$, and let $\mathbf{C}_{k}:=\tilde{\mathbf{D}}_{k}+\mathbf{L}_{k}$. If

(a) $0<\mathbf{D}_{k}=\mathbf{D}_{k}^{H} \lesssim \rho\left(\mathbf{M}_{k}^{-1} \mathbf{A}_{k}\right) \mathbf{M}_{k}$,

(b) $\mathbf{A}_{k} \leq \mathbf{D}_{k}+\mathbf{L}_{k}+\mathbf{L}_{k}^{H}$,

(c) $\left\|\mathbf{D}_{k}^{-\frac{1}{2}} \mathbf{L}_{k} \mathbf{D}_{k}^{-\frac{1}{2}}\right\| \lesssim 1$,

(d) $1-\left\|\mathbf{I}-\mathbf{D}_{k}^{\frac{1}{2}} \tilde{\mathbf{D}}_{k}^{-1} \mathbf{D}_{k}^{\frac{1}{2}}\right\| \gtrsim 1$, with $\|\cdot\|=\langle\cdot, \cdot\rangle^{\frac{1}{2}}$ being the Euclidean-norm, then (B) is valid.

Proof. From the definition of $\mathbf{C}_{k}, \mathrm{~b}$ and (2.15), it is sufficient to prove that

$$
\rho\left(\mathbf{M}_{k}^{-1} \mathbf{A}_{k}\right)\left(\tilde{\mathbf{D}}_{k}+\tilde{\mathbf{D}}_{k}^{H}-\mathbf{D}_{k}\right) \gtrsim\left(\tilde{\mathbf{D}}_{k}^{H}+\mathbf{L}_{k}\right) \mathbf{M}_{k}^{-1}\left(\tilde{\mathbf{D}}_{k}+\mathbf{L}_{k}\right)
$$

From

$$
\tilde{\mathbf{D}}_{k}+\tilde{\mathbf{D}}_{k}^{H}-\mathbf{D}_{k}=\tilde{\mathbf{D}}_{k}^{H} \mathbf{D}_{k}^{-\frac{1}{2}}\left(\mathbf{I}-\left(\mathbf{I}-\mathbf{D}_{k}^{\frac{1}{2}} \tilde{\mathbf{D}}_{k}^{-H} \mathbf{D}_{k}^{\frac{1}{2}}\right)\left(\mathbf{I}-\mathbf{D}_{k}^{\frac{1}{2}} \tilde{\mathbf{D}}_{k}^{-1} \mathbf{D}_{k}^{\frac{1}{2}}\right)\right) \mathbf{D}_{k}^{-\frac{1}{2}} \tilde{\mathbf{D}}_{k},
$$

which is valid for any invertible $\tilde{\mathbf{D}}_{k}$ and because $\mathbf{D}_{k}=\mathbf{D}_{k}^{H}>0$, we infer that

$$
\tilde{\mathbf{D}}_{k}+\tilde{\mathbf{D}}_{k}^{H}-\mathbf{D}_{k} \geq\left(1-\left\|\mathbf{I}-\mathbf{D}_{k}^{\frac{1}{2}} \tilde{\mathbf{D}}_{k}^{-1} \mathbf{D}_{k}^{\frac{1}{2}}\right\|^{2}\right) \tilde{\mathbf{D}}_{k}^{H} \mathbf{D}_{k}^{-1} \tilde{\mathbf{D}}_{k} .
$$


Because of this result together with $\mathrm{d}$, and because $\mathbf{M}_{k}^{-1} \lesssim \rho\left(\mathbf{M}_{k}^{-1} \mathbf{A}_{k}\right) \mathbf{D}_{k}^{-1}$ by 回 it is sufficient to show that

$$
\mathbf{D}_{k}^{-1} \gtrsim\left(\mathbf{I}+\tilde{\mathbf{D}}_{k}^{-H} \mathbf{L}_{k}\right) \mathbf{D}_{k}^{-1}\left(\mathbf{I}+\mathbf{L}_{k} \tilde{\mathbf{D}}_{k}^{-1}\right)
$$

The latter relation follows from

$$
\left\|\mathbf{D}_{k}^{-\frac{1}{2}}\left(\mathbf{I}+\mathbf{L}_{k} \tilde{\mathbf{D}}_{k}^{-1}\right) \mathbf{D}_{k}^{\frac{1}{2}}\right\| \leq 1+\left\|\mathbf{D}_{k}^{-\frac{1}{2}} \mathbf{L}_{k} \mathbf{D}_{k}^{-\frac{1}{2}}\right\|\left\|\mathbf{D}_{k}^{\frac{1}{2}} \tilde{\mathbf{D}}_{k}^{-1} \mathbf{D}_{k}^{\frac{1}{2}}\right\| \lesssim 1,
$$

by $\mathrm{C}$ and $\mathrm{d}$,

Remark 2.5. Condition d] of Proposition 2.4 means that $\tilde{\mathbf{D}}_{k}^{-1}$ is an approximate inverse of $\mathbf{D}_{k}$ which defines a uniformly convergent iteration in the "energy" norm $\left\langle\mathbf{D}_{k} \cdot, \cdot\right\rangle^{\frac{1}{2}}$.

Remark 2.6 ("inexact" point or block Gauss-Seidel). In the setting of Proposition 2.4, let $\mathbf{A}_{k}=\mathbf{D}_{k}+\mathbf{L}_{k}+\mathbf{L}_{k}^{H}$ be a partitioning of $\mathbf{A}_{k}$ into its (block) diagonal, its (block) lower triangular and its (block) upper triangular part, so that $\square$ is trivially valid.

Now $\mathbf{D}_{k}>0$ follows from $\mathbf{A}_{k}>0$. With $\Delta_{k}:=\operatorname{diag}\left(\mathbf{M}_{k}\right), \hat{\mathbf{A}}_{k}:=\Delta_{k}^{-\frac{1}{2}} \mathbf{A}_{k} \Delta_{k}^{-\frac{1}{2}}$, $\hat{\mathbf{D}}_{k}:=\Delta_{k}^{-\frac{1}{2}} \mathbf{D}_{k} \Delta_{k}^{-\frac{1}{2}}$ and $\hat{\mathbf{M}}_{k}:=\Delta_{k}^{-\frac{1}{2}} \mathbf{M}_{k} \Delta_{k}^{-\frac{1}{2}}$, the relation $\mathbf{D}_{k} \lesssim \rho\left(\mathbf{M}_{k}^{-1} \mathbf{A}_{k}\right) \mathbf{M}_{k}$ can be rewritten as

$$
\hat{\mathbf{D}}_{k} \lesssim \rho\left(\hat{\mathbf{M}}_{k}^{-1} \hat{\mathbf{A}}_{k}\right) \hat{\mathbf{M}}_{k}
$$

From $\hat{\mathbf{D}}_{k} \leq \rho\left(\hat{\mathbf{A}}_{k}\right) \mathbf{I} \leq \kappa\left(\hat{\mathbf{M}}_{k}\right) \rho\left(\hat{\mathbf{M}}_{k}^{-1} \hat{\mathbf{A}}_{k}\right) \hat{\mathbf{M}}_{k}$, a sufficient condition for $\mathbf{a}$ is $\kappa\left(\hat{\mathbf{M}}_{k}\right) \lesssim 1$. From

$$
\frac{\left\langle\mathbf{M}_{k} \mathbf{u}, \mathbf{u}\right\rangle}{\left\langle\Delta_{k} \mathbf{u}, \mathbf{u}\right\rangle}=\frac{\left\|\sum_{m \in I_{k}} \mathbf{u}_{k, m} \phi_{k, m}\right\|_{0, k}^{2}}{\sum_{m \in I_{k}}\left|\mathbf{u}_{k, m}\right|^{2}\left\|\phi_{k, m}\right\|_{0, k}^{2}}
$$

we see that $\kappa\left(\hat{\mathbf{M}}_{k}\right) \lesssim 1$ means uniform stability of the normalized bases of $V_{k}$ with respect to \|\|$_{0, k}$.

The Cauchy-Schwarz inequality $\left\langle\mathbf{A}_{k} \mathbf{u}, \mathbf{v}\right\rangle \mid \leq\left\langle\mathbf{A}_{k} \mathbf{u}, \mathbf{u}\right\rangle^{\frac{1}{2}}\left\langle\mathbf{A}_{k} \mathbf{v}, \mathbf{v}\right\rangle^{\frac{1}{2}}$ implies that all elements or blocks of $\mathbf{D}_{k}^{-\frac{1}{2}} \mathbf{L}_{k} \mathbf{D}_{k}^{-\frac{1}{2}}$ have absolute values or Euclidean norms less than or equal to one. So in case the number of elements or blocks, or more generally, the number of nonzero elements or blocks in each row and column is uniformly bounded, then $₫$ is valid. Applications are given by (inexact, i.e., $\tilde{\mathbf{D}}_{k} \neq \mathbf{D}_{k}$ ) point or block Gauss-Seidel iterations based on lexicographical or multicolor orderings of the unknowns.

Proposition 2.7. Let $\tilde{\mathbf{D}}_{k}, \mathbf{D}_{k}$ and $\mathbf{L}_{k}$ be some matrices of the same size as $\mathbf{A}_{k}$, and let $\mathbf{C}_{k}:=\rho\left(\mathbf{D}_{k}^{-1} \mathbf{A}_{k}\right) \tilde{\mathbf{D}}_{k}$. If

(a) $0<\mathbf{D}_{k}=\mathbf{D}_{k}^{H} \lesssim \frac{\rho\left(\mathbf{M}_{k}^{-1} \mathbf{A}_{k}\right)}{\rho\left(\mathbf{D}_{k}^{-1} \mathbf{A}_{k}\right)} \mathbf{M}_{k}$,

(b) $1-\left\|\mathbf{I}-\mathbf{D}_{k}^{\frac{1}{2}} \tilde{\mathbf{D}}_{k}^{-1} \mathbf{D}_{k}^{\frac{1}{2}}\right\| \gtrsim 1$,

then $(\mathrm{B})$ is valid.

Proof. In this case, (2.15) reads as

$$
\rho\left(\mathbf{M}_{k}^{-1} \mathbf{A}_{k}\right)\left(\tilde{\mathbf{D}}_{k}+\tilde{\mathbf{D}}_{k}^{H}-\rho\left(\mathbf{D}_{k}^{-1} \mathbf{A}_{k}\right)^{-1} \mathbf{A}_{k}\right) \gtrsim \rho\left(\mathbf{D}_{k}^{-1} \mathbf{A}_{k}\right) \tilde{\mathbf{D}}_{k}^{H} \mathbf{M}_{k}^{-1} \tilde{\mathbf{D}}_{k} .
$$

The proof follows from $\mathbf{A}_{k} \leq \rho\left(\mathbf{D}_{k}^{-1} \mathbf{A}_{k}\right) \mathbf{D}_{k}$, an application of (2.16), $\mathrm{b}$ and 
Remark 2.8 (preconditioned Richardson). If in Proposition [2.7] $\tilde{\mathbf{D}}_{k}=\omega^{-1} \mathbf{D}_{k}$ for some fixed $\omega \in \mathbb{R}$, then $\mathrm{b}$ means $\omega \in(0,2)$. Moreover, since after substituting $\tilde{\mathbf{D}}_{k}=\omega^{-1} \mathbf{D}_{k},(2.17)$ is equivalent to

$$
2 \omega \mathbf{I}-\omega^{2} \rho\left(\mathbf{D}_{k}^{-1} \mathbf{A}_{k}\right)^{-1} \mathbf{D}_{k}^{-\frac{1}{2}} \mathbf{A}_{k} \mathbf{D}_{k}^{-\frac{1}{2}} \gtrsim \frac{\rho\left(\mathbf{D}_{k}^{-1} \mathbf{A}_{k}\right)}{\rho\left(\mathbf{M}_{k}^{-1} \mathbf{A}_{k}\right)} \mathbf{D}_{k}^{\frac{1}{2}} \mathbf{M}_{k}^{-1} \mathbf{D}_{k}^{\frac{1}{2}},
$$

in this case both $\omega \in(0,2)$ and 国 are also necessary conditions for (B) .

Remark 2.9. Writing $\mathbf{A}_{k}-\mathbf{D}_{k}$ in the form $\mathbf{L}_{k}+\mathbf{L}_{k}^{H}$, condition 0 of Proposition 2.7 follows from $0<\mathbf{D}_{k} \lesssim \rho\left(\mathbf{M}_{k}^{-1} \mathbf{A}_{k}\right) \mathbf{M}_{k}$ and $\rho\left(\mathbf{D}_{k}^{-1} \mathbf{A}_{k}\right) \leq 1+2\left\|\mathbf{D}_{k}^{-\frac{1}{2}} \mathbf{L}_{k} \mathbf{D}_{k}^{-\frac{1}{2}}\right\| \lesssim 1$,

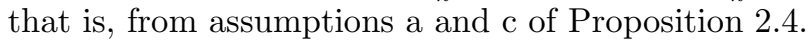

In particular, when $\mathbf{D}_{k}$ is a (block) diagonal part of $\mathbf{A}_{k}$, sufficient conditions for these assumptions are discussed in Remark 2.6 In this case, and with $\tilde{\mathbf{D}}_{k}=\omega^{-1} \mathbf{D}_{k}$, the iteration from Proposition 2.7 is known as the damped (block) Jacobi iteration with damping parameter $\rho\left(\mathbf{D}_{k}^{-1} \mathbf{A}_{k}\right)^{-1} \omega$. Iterations with $\tilde{\mathbf{D}}_{k}$ not equal to some multiple of $\mathbf{D}_{k}$ will be called inexact damped (block) Jacobi iterations.

2.5. The regularity and approximation assumption (A) with $\alpha=1$ in a nonconforming framework. We consider the following usual nonconforming finite element setting: Let

$$
\mathcal{H}^{2} \hookrightarrow \mathcal{H}^{1} \hookrightarrow \mathcal{H}^{0}
$$

be continuously embedded Hilbert spaces. We assume that, for all $k$,

$$
V_{k} \subset \mathcal{H}^{0}
$$

and put

$$
\|\cdot\|_{0, k}:=\|\cdot\|_{\mathcal{H}^{0}} \text {. }
$$

Furthermore, we assume that there exists a scalar product $a$ on $\mathcal{H}^{1}$ satisfying

$$
a(\cdot, \cdot)=\|\cdot\|_{\mathcal{H}^{1}}^{2}
$$

such that for all $k, a_{k}$ can be extended to a scalar product on $\mathcal{H}^{1}+V_{k}$, which reduces to $a$ on $\mathcal{H}^{1}$. Finally, we assume that the sequence $\left(\rho_{k}\right)_{k}$ defined in (2.8) satisfies $\rho_{k+1} \lesssim \rho_{k}$.

Theorem 2.10. For $f \in \mathcal{H}^{0}$, let $u \in \mathcal{H}^{1}$ denote the solution of

$$
a(u, v)=(f, v)_{\mathcal{H}^{0}} \quad\left(v \in \mathcal{H}^{1}\right) .
$$

Then, if

(a) $u \in \mathcal{H}^{2}$ with $\|u\|_{\mathcal{H}^{2}} \lesssim\|f\|_{\mathcal{H}^{0}} \quad$ ("full" regularity),

(b) $\left|a_{k}\left(u, v_{k}\right)-\left(f, v_{k}\right)_{\mathcal{H}^{0}}\right| \lesssim \rho_{k}^{-\frac{1}{2}} a_{k}\left(v_{k}, v_{k}\right)^{\frac{1}{2}}\|f\|_{\mathcal{H}^{0}} \quad\left(v_{k} \in \mathcal{H}^{1}+V_{k}\right)$ (consistency)

(c) $\inf _{v_{k} \in V_{k}} a_{k}\left(v-v_{k}, v-v_{k}\right)^{\frac{1}{2}} \lesssim \rho_{k}^{-\frac{1}{2}}\|v\|_{\mathcal{H}^{2}} \quad\left(v \in \mathcal{H}^{2}\right)$ (approximation)

and there exist mappings $\Pi_{k}: \mathcal{H}^{2} \rightarrow V_{k}$ such that

(d) $\left\|I-\Pi_{k}\right\|_{\mathcal{H}^{0} \leftarrow \mathcal{H}^{2}} \lesssim \rho_{k}^{-1}$

(e) $\left\|\Pi_{k}-I_{k} \Pi_{k-1}\right\|_{\mathcal{H}^{0} \leftarrow \mathcal{H}^{2}} \lesssim \rho_{k}^{-1}$ and finally,

(f) $\left\|I_{k}\right\|_{\mathcal{H}^{0} \leftarrow \mathcal{H}^{0}} \lesssim 1$, 
then

$$
\left|a_{k}\left(\left(I-I_{k} I_{k}^{*}\right) v_{k}, v_{k}\right)\right| \lesssim \rho_{k}^{-1}\left\|v_{k}\right\|_{2, k}^{2} \quad\left(v_{k} \in V_{k}\right)
$$

(Assumption (A) with $\alpha=1$ ).

Proof. By the definition of \|\|$_{2, k}$ in (2.7), it follows that

$$
\left|a_{k}\left(w_{k}, v_{k}\right)\right| \leq\left\|w_{k}\right\|_{\mathcal{H}^{0}}\left\|v_{k}\right\|_{2, k} \quad\left(w_{k}, v_{k} \in V_{k}\right),
$$

which means that it is sufficient to show that

$$
\left\|\left(I-I_{k} I_{k}^{*}\right) v_{k}\right\|_{\mathcal{H}^{0}}=\sup _{0 \neq f \in \mathcal{H}^{0}} \frac{\left|\left(f,\left(I-I_{k} I_{k}^{*}\right) v_{k}\right)_{\mathcal{H}^{0}}\right|}{\|f\|_{\mathcal{H}^{0}}} \lesssim \rho_{k}^{-1}\left\|v_{k}\right\|_{2, k} \quad\left(u_{k} \in V_{k}\right) .
$$

Given $f \in \mathcal{H}^{0}$, we define $u \in \mathcal{H}^{1}$ and, for each $k, u_{k} \in V_{k}$ by

$$
\begin{aligned}
a(u, w)=(f, w)_{\mathcal{H}^{0}} & \left(w \in \mathcal{H}^{1}\right), \\
a_{k}\left(u_{k}, w_{k}\right)=\left(f, w_{k}\right)_{\mathcal{H}^{0}} & \left(w_{k} \in V_{k}\right) .
\end{aligned}
$$

From

$$
\begin{aligned}
\left.\left(f,\left(I-I_{k} I_{k}^{*}\right) v_{k}\right)\right)_{\mathcal{H}^{0}} & =a_{k}\left(u_{k},\left(I-I_{k} I_{k}^{*}\right) v_{k}\right) \\
& =a_{k}\left(u_{k}-I_{k} u_{k-1}, v_{k}\right)+a_{k}\left(I_{k}\left(u_{k-1}-I_{k}^{*} u_{k}\right), v_{k}\right),
\end{aligned}
$$

together with (2.18) and ff we see that (2.19) will follow from

$$
\begin{aligned}
\left\|u_{k}-I_{k} u_{k-1}\right\|_{\mathcal{H}^{0}} & \lesssim \rho_{k}^{-1}\|f\|_{\mathcal{H}^{0}}, \\
\left\|u_{k-1}-I_{k}^{*} u_{k}\right\|_{\mathcal{H}^{0}} & \lesssim \rho_{k}^{-1}\|f\|_{\mathcal{H}^{0}} .
\end{aligned}
$$

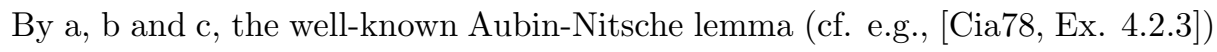
shows that

$$
\left\|u-u_{k}\right\|_{\mathcal{H}^{0}} \lesssim \rho_{k}^{-1}\|f\|_{\mathcal{H}^{0}} \quad \text { (convergence) }
$$

By writing

$$
u_{k}-I_{k} u_{k-1}=\left(u_{k}-\Pi_{k} u\right)+\left(\Pi_{k} u-I_{k} \Pi_{k-1} u\right)+I_{k}\left(\Pi_{k-1} u-u_{k-1}\right),
$$

(2.20) follows from (2.22), 囵 따 1 and

To establish (2.21), we write

$$
\left\|u_{k-1}-I_{k}^{*} u_{k}\right\|_{\mathcal{H}^{0}}=\sup _{0 \neq g \in \mathcal{H}^{0}} \frac{\left|\left(g, u_{k-1}-I_{k}^{*} u_{k}\right)_{\mathcal{H}^{0}}\right|}{\|g\|_{\mathcal{H}^{0}}},
$$

and, for given $g \in \mathcal{H}^{0}$, we define $z \in \mathcal{H}^{1}$ and, for each $k, z_{l} \in V_{l}$ by

$$
\begin{aligned}
a(z, w)=(g, w)_{\mathcal{H}^{0}} & \left(w \in \mathcal{H}^{1}\right), \\
a_{k}\left(z_{k}, w_{k}\right)=\left(g, w_{k}\right)_{\mathcal{H}^{0}} & \left(w_{k} \in V_{k}\right) .
\end{aligned}
$$

By writing

$$
\begin{aligned}
\left(g, u_{k-1}-I_{k}^{*} u_{k}\right)_{\mathcal{H}^{0}} & =\left(g, u_{k-1}\right)_{\mathcal{H}^{0}}-a_{k-1}\left(z_{k-1}, I_{k}^{*} u_{k}\right) \\
& =\left(g, u_{k-1}\right)_{\mathcal{H}^{0}}-a_{k}\left(I_{k} z_{k-1}, u_{k}\right) \\
& =\left(g, u_{k-1}-u_{k}\right)_{\mathcal{H}^{0}}+a_{k}\left(z_{k}-I_{k} z_{k-1}, u_{k}\right) \\
& =\left(g, u_{k-1}-u_{k}\right)_{\mathcal{H}^{0}}+\left(z_{k}-I_{k} z_{k-1}, f\right)_{\mathcal{H}^{0}},
\end{aligned}
$$

(2.21) follows from (2.22) and (2.20). 
Remark 2.11. Under the assumptions of Theorem 2.10, most proofs from the literature yield assumption (A) only for $\alpha=\frac{1}{2}$. An exception is Bre99, which our proof is partly based upon. Yet, compared to that paper, our proof is shorter and needs fewer assumptions. On the other hand, the arguments from Bre99 are not restricted to the "full" regularity case. In view of the multi-grid convergence theory from Section 2.3 it is desirable to have (A) with $\alpha$ as high as possible, and in particular to know whether it is valid for $\alpha=1$.

In [BDH99, §4] a result similar to Theorem 2.10 was proved. Instead of $\square$ and $\mathrm{C}$ there (2.22) was assumed, which is clearly also a sufficient condition for the present

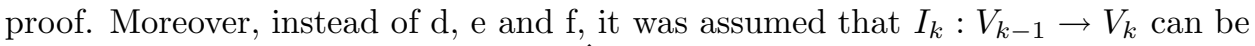
extended to an $\mathcal{H}^{0}$-bounded projector $\hat{I}_{k}$ from $V_{k-1}+V_{k}$ onto $V_{k}$. Obviously, (2.20) can also be deduced from this property, which means that our proof applies as well. Although in applications often the condition involving $\hat{I}_{k}$ is more easily verified, in connection with the Morley element we will encounter an $I_{k}$ of practical interest for which $\mathrm{d}, \mathrm{a}$ and $f$ are valid, but the condition involving $\hat{I}_{k}$ is not.

2.6. Additive multi-grid method. In particular to underline the role of the iterated prolongations in the behaviour of multi-grid methods, we briefly discuss the additive variant. Given some scalar products $e_{k}$ on $V_{k}$, we define $E_{k}: V_{k} \rightarrow V_{k}^{\prime}$, determining a Hermitian smoother, by

$$
\left(E_{k} u\right)(v)=e_{k}(u, v) \quad\left(u, v \in V_{k}\right) .
$$

The additive multi-grid operator $B_{k}^{(\text {add) }}$ is now defined by

$$
B_{k}^{(\text {add })}=\left\{\begin{array}{cc}
E_{k}^{-1}+I_{k} B_{k-1}^{(\text {add })} I_{k}^{\prime} & \text { if } k>0, \\
A_{0}^{-1} & \text { if } k=0 .
\end{array}\right.
$$

The following result can be deduced from Osw97]:

Theorem 2.12. For $k \geq 0$, let $P_{k}: V_{k+1} \rightarrow V_{k}$ be some mappings. Put

$$
I_{j \leftarrow k}:=\left\{\begin{array}{cl}
I_{j} I_{j-1 \leftarrow k} & \text { if } j>k, \\
I & \text { if } j=k,
\end{array} \text { and } P_{k \leftarrow j}:=\left\{\begin{array}{cl}
P_{k \leftarrow j-1} P_{j-1} & \text { if } j>k, \\
I & \text { if } j=k .
\end{array}\right.\right.
$$

Then, with $I_{0} P_{-1}:=0$,

$$
\begin{aligned}
& \inf _{0 \neq u \in V_{j}} \frac{e_{j}(u, u)}{a_{j}(u, u)} \cdot \max _{0 \leq k \leq j} \sup _{0 \neq u \in V_{k}} \frac{a_{j}\left(I_{j \leftarrow k} u, I_{j \leftarrow k} u\right)}{e_{k}(u, u)}
\end{aligned}
$$

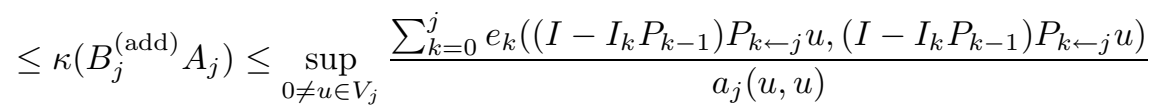

$$
\begin{aligned}
& \cdot \sum_{k=0}^{j} \sup _{0 \neq u \in V_{k}} \frac{a_{j}\left(I_{j \leftarrow k} u, I_{j \leftarrow k} u\right)}{e_{k}(u, u)} .
\end{aligned}
$$

In particular, if

$$
a_{k}(u, u) \lesssim e_{k}(u, u) \lesssim \rho_{k}\|u\|_{0, k}^{2} \quad\left(u \in V_{k}\right)
$$

then with

$$
t_{j}:=\sup _{0 \neq u \in V_{j}} \frac{\sum_{k=0}^{j} \rho_{k}\left\|\left(I-I_{k} P_{k-1}\right) P_{k \leftarrow j} u\right\|_{0, k}^{2}}{a_{j}(u, u)}
$$


it follows that

$$
\max _{0 \leq k \leq j} \sup _{0 \neq u \in V_{k}} \frac{a_{j}\left(I_{j \leftarrow k} u, I_{j \leftarrow k} u\right)}{e_{k}(u, u)} \lesssim \kappa\left(B_{j}^{\text {(add) }} A_{j}\right) \lesssim t_{j} \sum_{k=0}^{j} \rho\left(I_{j \leftarrow k}^{*} I_{j \leftarrow k}\right) .
$$

Remark 2.13. Let us assume (2.23). In our applications, it will appear that the $P_{k}$ can be selected such that either $t_{j} \lesssim 1$ or $t_{j} \lesssim j$, and so $\kappa\left(B_{j}^{\text {(add) }} A_{j}\right) \lesssim$ $j \max _{0 \leq k \leq j} \rho\left(I_{j \leftarrow k}^{*} I_{j \leftarrow k}\right)$ or $\kappa\left(B_{j}^{\text {(add })} A_{j}\right) \lesssim j^{2} \max _{0 \leq k \leq j} \rho\left(I_{j \leftarrow k}^{*} I_{j \leftarrow k}\right)$. On the other hand, for any fixed $k$, based on $a_{k}(,) \equiv e_{k}($,$) , we have \kappa\left(B_{j}^{\text {(add) }} A_{j}\right) \gtrsim \rho\left(I_{j \leftarrow k}^{*} I_{j \leftarrow k}\right)$ $(j \geq k)$. Together these bounds show that the quality of the additive multi-grid preconditioner depends critically on the energy norm of the iterated prolongations.

Recall that at the end of Subsection [2.3] for $B_{j}$ defined by the (multiplicative) standard $\mathrm{V}$-cycle, we observed that the upper bound on $\kappa\left(B_{j} A_{j}\right)$ depends critically on the energy norm of the "iterated prolongations alternated with smoothers", i.e., on the factors $\rho\left(\tilde{I}_{j \leftarrow k}^{*} \tilde{I}_{j \leftarrow k}\right)$. Since by assumption (B),$\rho\left(K_{k}^{*} K_{k}\right) \leq 1$, it is likely that $\rho\left(I_{j \leftarrow k}^{*} I_{j \leftarrow k}\right) \lesssim 1$ would imply that $\rho\left(\tilde{I}_{j \leftarrow k}^{*} \tilde{I}_{j \leftarrow k}\right) \lesssim 1$. On the other hand, if for example $\rho\left(I_{j \leftarrow k}^{*} I_{j \leftarrow k}\right)$ is an exponentially increasing function of $j-k$, then for general smoothers it cannot be expected that $\rho\left(\tilde{I}_{j \leftarrow k}^{*} \tilde{I}_{j \leftarrow k}\right) \lesssim 1$.

Remark 2.14. Even when $t_{j} \lesssim 1$ and $\rho\left(I_{j \leftarrow k}^{*} I_{j \leftarrow k}\right) \lesssim 1$, Theorem 2.12 only shows that the additive multi-grid preconditioner is suboptimal, i.e., $\kappa\left(B_{j}^{(\text {add })} A_{j}\right) \lesssim j$. Yet, under the conditions that were imposed, this is the best result one can expect. Indeed, consider the case that $0 \neq V_{0} \subset \cdots \subset V_{j-1} \subsetneq V_{j}$, for all $1 \leq k \leq j$, $e_{k}=a_{k}=a_{j}$, and $I_{k}$ is the trivial injection. It is not difficult to show that then $\kappa\left(B_{j}^{(\text {add })} A_{j}\right)=j+1$.

Below we comment on the construction of Hermitian smoothers that satisfy (2.23). If the forms $c_{k}$ introduced in Section 2.1 are Hermitian and (B) is valid, then the equivalence of (B) and (2.14) shows that $2 c_{k}(u, u)>a_{k}(u, u)$ and $c_{k}(u, u) \lesssim$ $\rho_{k}\|u\|_{0, k}^{2}$, or $e_{k}=c_{k}$ satisfies (2.23).

The reverse is not valid; taking $c_{k}=e_{k}$, where $e_{k}$ satisfies (2.23), does not imply $(\mathrm{B})$. Indeed, note for example that (2.23) does not guarantee that $2 e_{k}>a_{k}$, that is, convergence of the corresponding iteration.

In case $c_{k}$ is non-Hermitian, an obvious candidate for a suitable Hermitian smoother is the symmetrized smoother defined as follows: Let $C_{k}, C_{k}^{\dagger}$ be defined as in (2.4). For $g \in V_{k}^{\prime}$, put $G_{k} g=x^{(2)}$, where $x^{(0)}=0$ and

$$
\left\{\begin{array}{l}
x^{(1)}=x^{(0)}+C_{k}^{-1}\left(g-A_{k} x^{(0)}\right), \\
x^{(2)}=x^{(1)}+C_{k}^{-\dagger}\left(g-A_{k} x^{(1)}\right),
\end{array}\right.
$$

or $G_{k}=C_{k}^{-1}+C_{k}^{-\dagger}-C_{k}^{-\dagger} A_{k} C_{k}^{-1}$.

Proposition 2.15. $E_{k}:=G_{k}^{-1}$ exists, and $e_{k}(u, v)=\left(E_{k} u\right)(v)$ are scalar products that satisfy (2.23) if and only if the $c_{k}$ satisfy (B).

Proof. Assumption (B) can be rewritten as

$$
a_{k}\left(G_{k} A_{k} u, u\right) \gtrsim \rho_{k}^{-1}\|u\|_{2, k}^{2} \quad\left(u \in V_{k}\right),
$$

which shows that $E_{k}$ exists. 
From $a_{k}\left(\left(I-G_{k} A_{k}\right) u, u\right)=a_{k}\left(K_{k}^{*} K_{k} u, u\right) \geq 0$, we have $a_{k}\left(G_{k} A_{k} u, u\right) \leq a_{k}(u, u)$, or, by substituting $u=\left(G_{k} A_{k}\right)^{-\frac{1}{2}} v$,

$$
a_{k}(v, v) \leq a_{k}\left(A_{k}^{-1} E_{k} v, v\right)=e_{k}(v, v) \quad\left(v \in V_{k}\right) .
$$

Substituting $u=\left(G_{k} A_{k}\right)^{-1} w$ in (2.25) yields

$$
e_{k}(w, w) \gtrsim \rho_{k}^{-1} \sup _{0 \neq v \in V_{k}} \frac{\left|e_{k}(w, v)\right|^{2}}{\|v\|_{0, k}^{2}},
$$

and so, by taking $v=w$, in particular

$$
e_{k}(w, w) \lesssim \rho_{k}\|w\|_{0, k}^{2} \quad\left(w \in V_{k}\right) .
$$

Together, formulas (2.26) and (2.28) show that (2.23) is valid.

On the other hand, if $e_{k}(u, v):=\left(E_{k} u\right)(v)$ are scalar products that satisfy (2.23) and thus (2.28), then by an application of the Cauchy-Schwarz inequality we infer (2.27) and so (2.25), that is, $(\mathrm{B})$ is valid.

\section{Applications}

3.1. Nonconforming $P_{1}$ element. Let $\tau_{0}, \tau_{1}, \ldots$ be a sequence of conforming triangulations of some bounded, convex polygon $\Omega \subset \mathbb{R}^{2}$, such that $\tau_{k+1}$ is generated from $\tau_{k}$ by refinement, $\sup _{T \in \tau_{k}} \operatorname{diam}(T) \approx 2^{-k}$, and the triangles satisfy a shape regularity condition uniformly over the levels. We define $\bar{E}_{k}$ and $E_{k}$ as the sets of all and of all internal edges of $\tau_{k}$, respectively. For $e \in \bar{E}_{k}, m_{e}$ will denote the midpoint of $e$. We take $V_{k}=V_{k}^{\left(P_{1}\right)}$, where

$$
\begin{array}{r}
V_{k}^{\left(P_{1}\right)}=\left\{v \in \prod_{T \in \tau_{k}} P_{1}(T): v \text { is continuous at } m_{e} \text { for } e \in E_{k},\right. \\
\text { and it vanishes at } \left.m_{e} \text { for } e \in \bar{E}_{k} \backslash E_{k}\right\},
\end{array}
$$

and define

$$
a_{k}(u, v)=\sum_{T \in \tau_{k}} \int_{T} \nabla u \cdot \nabla v
$$

With \|\|$_{0, k}:=\|\|_{L^{2}(\Omega)}$, we find that $\rho_{k}$ defined in (2.8) satisfies $\rho_{k} \approx 4^{k}$.

We define the prolongation in the usual way, that is,

$$
\left(I_{k} u\right)\left(m_{e}\right)=\text { average }_{i} \text { of }\left.u\right|_{T_{i}}\left(m_{e}\right) \quad\left(e \in E_{k}\right),
$$

where $\tau_{k-1} \ni T_{i} \supset e$.

In the setting of Section 2.5, let

$$
\mathcal{H}^{0}=L^{2}(\Omega), \quad \mathcal{H}^{1}=H_{0}^{1}(\Omega), \quad \mathcal{H}^{2}=H^{2}(\Omega) \cap H_{0}^{1}(\Omega),
$$

and

$$
a(u, v)=\int_{\Omega} \nabla u \cdot \nabla v .
$$

With these definitions, it is well-known that conditionsarc of Theorem 2.10 are satisfied (for b and $\mathrm{C}$ see, e.g., [BS94, §8.3]). We define $\Pi_{k}: H^{2}(\Omega) \cap H_{0}^{1}(\Omega) \rightarrow V_{k}^{\left(P_{1}\right)}$ by $\left(\Pi_{k} u\right)\left(m_{e}\right)=u\left(m_{e}\right)$. Then, using the local reproduction by $I_{k}$ of first degree polynomials, standard arguments like the Sobolev embedding theorem and the Bramble-Hilbert lemma show the remaining conditions $\mathrm{c}$, $\mathrm{d}$ and $f$ From Theorem [2.10 we conclude that assumption (A) with $\alpha=1$ is valid. 
We now equip the spaces $V_{k}^{\left(P_{1}\right)}$ with nodal bases $\left\{\eta_{k, e}: e \in E_{k}\right\}$, defined by

$$
\eta_{k, e}\left(m_{\tilde{e}}\right)=\delta_{e, \tilde{e}} \quad\left(e, \tilde{e} \in E_{k}\right) .
$$

These bases are $L^{2}(\Omega)$-orthogonal, and so, as demonstrated in Section 2.4, (inexact) standard Gauss-Seidel and damped Jacobi smoothers satisfy assumption (B).

Since $(\mathrm{A}$ ) and $(\mathrm{B})$ are valid, with a number of smoothing steps $m$ that is sufficiently large, the W-cycle yields uniformly convergent iterations. Yet, since generally $\rho\left(I_{k}^{*} I_{k}\right)>2$, which has been demonstrated in [Che97, Ex.1], for any $m$ that happens not to be large enough the $\mathrm{W}$-cycle might result in a preconditioned system that is indefinite. On the other hand, the variable $\mathrm{V}$-cycle yields preconditioned systems that have uniformly bounded condition numbers. Since (A) is valid with $\alpha=1$, Theorem 2.3 even shows that this also holds for the mildly variable $\mathrm{V}$-cycle.

We now consider the additive multi-grid method. By taking $P_{k}$ to be the restriction to $V_{k+1}^{\left(P_{1}\right)}$ of the $a_{k+1}($,$) -orthogonal projector from V_{k+1}^{\left(P_{1}\right)}+V_{k}^{\left(P_{1}\right)}$ to $V_{k}^{\left(P_{1}\right)}$, Osw97 has proved that the scalars $t_{j}$ from Theorem 2.12 are uniformly bounded. Furthermore, assuming uniform dyadic refinements and under some technical assumptions concerning the degree of the nodes in the mesh, in Osw92] it was shown that $\rho\left(I_{j \leftarrow k}^{*} I_{j \leftarrow k}\right) \lesssim 1$. Assuming a Hermitian smoother that satisfies (2.23), from Theorem 2.12 we conclude that the additive multi-grid preconditioner $B_{j}^{(\text {add) }}$ satisfies $\kappa\left(B_{j}^{(\text {add })} A_{j}\right) \lesssim j$. Since regularity plays no role in the analysis of the additive method, this result is also valid for nonconvex $\Omega$.

Because $\rho\left(I_{j \leftarrow k}^{*} I_{j \leftarrow k}\right) \lesssim 1$, it is likely that also $\rho\left(\tilde{I}_{j \leftarrow k}^{*} \tilde{I}_{j \leftarrow k}\right) \lesssim 1$. According to the observations made at the end of Section 2.3, this would mean that the standard (multiplicative) $\mathrm{V}$-cycle yields a preconditioner $B_{j}$ for which at least $\kappa\left(B_{j} A_{j}\right) \lesssim j$.

3.2. Rotated $Q_{1}$ element. Now let $\tau_{0}, \tau_{1}, \ldots$ be a sequence of conforming subdivisions of some bounded, convex polygon $\Omega \subset \mathbb{R}^{2}$ into parallelograms, such that $\tau_{k+1}$ is generated from $\tau_{k}$ by refinement, $\sup _{T \in \tau_{k}} \operatorname{diam}(T) \equiv 2^{-k}$, and the parallelograms satisfy a shape regularity condition uniformly over the levels. We define $\bar{E}_{k}$ and $E_{k}$ as the sets of all or internal edges of $\tau_{k}$, respectively. For $e \in \bar{E}_{k}, m_{e}$ will denote the midpoint of $e$. For each $T \in \tau_{k}$, we consider the space $P_{T}=\left\{v \in L^{2}(T): v \circ F_{T} \in \operatorname{span}\left\{1, x, y, x^{2}-y^{2}\right\}\right\}$, where $F_{T}$ is an affine bijection between $[-1,1]^{2}$ and $T$. There are two usual options to identify $v \in P_{T}$ uniquely, namely either by

$$
\left\{v\left(m_{e}\right): e \in E_{k}\right\}
$$

or by

$$
\left\{\frac{1}{|e|} \int_{e} v: e \in E_{k}\right\}
$$

Both choices give rise to different finite element spaces $V_{k}=V_{k}^{\left(Q_{1}\right)}$ defined by

$$
\begin{array}{r}
V_{k}^{\left(Q_{1}\right)}=\left\{v \in \prod_{T \in \tau_{k}} P_{T}: \text { the degrees of freedom match at } e \in E_{k},\right. \\
\text { and they vanish at } \left.e \in \bar{E}_{k} \backslash E_{k}\right\},
\end{array}
$$

Similarly to Section 3.1 we take

$$
a_{k}(u, v)=\sum_{T \in \tau_{k}} \int_{T} \nabla u \cdot \nabla v
$$

and with \|\|$_{0, k}:=\|\|_{L^{2}(\Omega)}$, we find that $\rho_{k}$ defined in (2.8) satisfies $\rho_{k} \bar{\approx} 4^{k}$. 
As in Section 3.1 the prolongations $I_{k}$ can be defined by averaging the degrees of freedom at $e \in E_{k}$, and by setting them equal to zero at $e \in \bar{E}_{k} \backslash E_{k}$. For both (3.2) and (3.3), the resulting $I_{k}$ reproduces first degree polynomials.

With $\mathcal{H}^{0}, \mathcal{H}^{1}, \mathcal{H}^{2}$ and $a($,$) as in Section [3.1, all conditions of Theorem [2.10] can$ be verified analogously. We conclude that assumption (A) with $\alpha=1$ is valid.

Since for both (3.2) and (3.3), the normalized bases of $V_{k}^{\left(Q_{1}\right)}$ with respect to the degrees of freedom are uniformly $L^{2}(\Omega)$-stable, from Section 2.4 we learn that (inexact) standard Gauss-Seidel and damped Jacobi smoothers satisfy (B). We conclude that the $\mathrm{W}$-cycle with a sufficiently large number of smoothing iterations, the variable $\mathrm{V}$-cycle and even the mildly variable $\mathrm{V}$-cycle all yield uniformly convergent iterations or preconditioned systems with uniformly bounded condition numbers.

Assuming that $\tau_{k}$ corresponds to a uniform partition of $\Omega$ into squares, for the choice [3.3), in CO98] it was shown that $\rho\left(I_{k}^{*} I_{k}\right) \leq 2$ (but generally $\rho\left(I_{k}^{*} I_{k}\right)>1$, see [Che97]). This means that even for any positive number of smoothing iterations the $\mathrm{W}$-cycle yields uniformly convergent iterations.

Again for (3.3), and under the same assumption on the mesh, in CO98 it was shown that $\rho\left(I_{j \leftarrow k}^{*} I_{j \leftarrow k}\right) \lesssim 1$. Since, for $P_{k}$ being the restriction to $V_{k+1}^{\left(Q_{1}\right)}$ of the $a_{k+1}($,$) -orthogonal projector from V_{k+1}^{\left(Q_{1}\right)}+V_{k}^{\left(Q_{1}\right)}$ to $V_{k}^{\left(Q_{1}\right)}$, it was proved that $t_{j} \lesssim 1$, Theorem 2.12 now shows that with a suitable Hermitian smoother the additive multi-grid preconditioner is suboptimal, i.e., $\kappa\left(B_{j}^{(\text {add })} A_{j}\right) \lesssim j$. Since it is likely that as a consequence also $\rho\left(\tilde{I}_{j \leftarrow k}^{*} \tilde{I}_{j \leftarrow k}\right) \lesssim 1$, this would mean that the standard (multiplicative) V-cycle yields a preconditioner that is at least suboptimal.

On the other hand, for the choice (3.2), we know that $\rho\left(I_{k}^{*} I_{k}\right)>2$ ([Che97]). This means that with a number of smoothing steps that is not large enough, the $\mathrm{W}$-cycle might result in a preconditioned system that is indefinite. Moreover, again for (3.2), numerical results from Osw97] indicate that for fixed $k, \rho\left(I_{j \leftarrow k}^{*} I_{j \leftarrow k}\right)$ increases exponentially as a function of $j-k$. By Remark 2.13 , this means that also $\kappa\left(B_{j}^{\text {(add) }} A_{j}\right)$ is an exponentially growing function of $j \geq k$. Moreover, since in this case for general smoothers it cannot be expected that $\rho\left(\tilde{I}_{j \leftarrow k}^{*} \tilde{I}_{j \leftarrow k}\right) \lesssim 1$, the discussion at the end of Section 2.3 shows that the standard (multiplicative) $\mathrm{V}$-cycle might give unsatisfactory results as well.

\subsection{Morley element.}

3.3.1. The discretized biharmonic equation. Let $\tau_{0}, \tau_{1}, \ldots$ be a sequence of conforming triangulations of some bounded, convex polygon $\Omega \subset \mathbb{R}^{2}$, such that $\tau_{k+1}$ is generated from $\tau_{k}$ by refinement, $\sup _{T \in \tau_{k}} \operatorname{diam}(T) \stackrel{\equiv}{\sim} 2^{-k}$, and the triangles satisfy a shape regularity condition uniformly over the levels. We define $\bar{E}_{k}, \bar{N}_{k}$ as the set of all edges and vertices of $\tau_{k}$, and $E_{k}, N_{k}$ as the set of internal edges and vertices of $\tau_{k}$. For $e \in \bar{E}_{k}, m_{e}$ will denote the midpoint of $e$, and $\mathbf{n}_{e}$ a unit vector normal to $e$. We take $V_{k}=M_{k}$, where $M_{k}$ is the Morley finite element space corresponding to $\tau_{k}$, i.e.,

$$
\begin{array}{r}
M_{k}=\left\{v \in \prod_{T \in \tau_{k}} P_{2}(T): v \text { is continuous at } p \in N_{k} \text { and vanishes at } p \in \bar{N}_{k} \backslash N_{k} ;\right. \\
\left.\partial_{\mathbf{n}_{e}} v \text { is continuous at } m_{e} \text { for } e \in E_{k} \text { and vanishes at } m_{e} \text { for } e \in \bar{E}_{k} \backslash E_{k}\right\} .
\end{array}
$$

Since $v \in M_{k}$ is piecewise quadratic, its derivative tangential to $e \in \bar{E}_{k}$ in $m_{e}$ from either side of $e$ (if $e \in E_{k}$ ) can be expressed as a divided difference in terms of 
the values of $v$ at the endpoints of $e$. The continuity of $v$ at the vertices therefore shows that also these tangential derivatives are continuous for $e \in E_{k}$ and vanish for $e \in \bar{E}_{k} \backslash E_{k}$.

We define $a_{k}=a_{k}^{(\mathrm{Bih})}$ by

$$
a_{k}^{(\mathrm{Bih})}(u, v):=\sum_{T \in \tau_{k}} \int_{T} \sum_{i, j=1}^{2} \frac{\partial^{2} u}{\partial x_{i} \partial x_{j}} \frac{\overline{\partial^{2} v}}{\partial x_{i} \partial x_{j}} .
$$

The prolongation $I_{k}^{(\mathrm{Bih}, 1)}: M_{k-1} \rightarrow M_{k}$ commonly used in connection with the Morley finite element spaces was introduced in [Bre89], and is defined by

$$
\begin{aligned}
\left(I_{k}^{(\mathrm{Bih}, 1)} u\right)(p) & =\text { average }_{i} \text { of }\left.u\right|_{T_{i}}(p) \quad\left(p \in N_{k}\right), \\
\partial_{\mathbf{n}_{e}}\left(I_{k}^{(\mathrm{Bih}, 1)} u\right)\left(m_{e}\right) & =\text { average }_{i} \text { of } \partial_{\mathbf{n}_{e}}\left(\left.u\right|_{T_{i}}\right)\left(m_{e}\right) \quad\left(e \in E_{k}\right),
\end{aligned}
$$

where $\tau_{k-1} \ni T_{i} \ni p$ or $\tau_{k-1} \ni T_{i} \supset e$.

As appears from numerical results reported in Osw97, Che97, a disadvantage of $I_{k}^{(\mathrm{Bih}, 1)}$ is that, for fixed $k, \rho\left(\left(I_{j \leftarrow k}^{(\mathrm{Bih}, 1)}\right)^{*} I_{j \leftarrow k}^{(\mathrm{Bih}, 1)}\right)$ generally grows exponentially with $j-k$. As we said before, this has an adverse effect on the additive and (V-cycle type) multiplicative multi-grid methods.

In this paper, we therefore introduce an alternative prolongation $I_{k}^{(\mathrm{Bih}, 2)}$. For ease of presentation, we restrict ourselves to the case of uniform dyadic refinements. Let $E_{k}^{(\text {new })}$ denote the set of new edges, i.e., $E_{k}^{(\text {new })}=\left\{e \in E_{k}: e \not \subset \tilde{e}\right.$ for any $\tilde{e} \in$ $\left.E_{k-1}\right\}$. Then $I_{k}^{(\mathrm{Bih}, 2)}$ is defined by

$$
\left(I_{k}^{(\mathrm{Bih}, 2)} u\right)(p)=\text { average }_{i} \text { of }\left.u\right|_{T_{i}}(p) \quad\left(p \in N_{k}\right),
$$

where $\tau_{k-1} \ni T_{i} \ni p$,

$$
\partial_{\mathbf{n}_{e}}\left(I_{k}^{(\mathrm{Bih}, 2)} u\right)\left(m_{e}\right)=\partial_{\mathbf{n}_{e}} u\left(m_{e}\right) \quad\left(e \in E_{k}^{(\text {new })}\right)
$$

and

$$
a_{k}^{(\mathrm{Bih})}\left(I_{k}^{(\mathrm{Bih}, 2)} u, \check{M}_{k}\right)=0,
$$

where $\check{M}_{k}$ is defined as the span of the remaining degrees of freedom, i.e.,

$$
\check{M}_{k}=\left\{u \in M_{k}: u\left(N_{k}\right)=0, \partial_{\mathbf{n}_{e}} u\left(m_{e}\right)=0\left(e \in E_{k}^{(\text {new })}\right)\right\} .
$$

Note that (3.5)-(3.6) coincide with the corresponding definitions of $I_{k}^{(\mathrm{Bih}, 1)}$, and that for each $\tilde{p} \in N_{k-1}$, equation (3.7) involves solving a small system with unknowns the values $\partial_{\mathbf{n}_{e}}\left(I_{k}^{(\mathrm{Bih}, 2)} u\right)\left(m_{e}\right)$ for all edges $e \in E_{k} \backslash E_{k}^{(\text {new })}$ that contain $\tilde{p}$ (see Figure 1).

Since among all prolongations $I_{k}^{(\mathrm{Bih})}: M_{k-1} \rightarrow M_{k}$ satisfying (3.5)-3.6), $I_{k}^{(\mathrm{Bih}, 2)}$ is the one for which $a_{k}\left(I_{k}^{(\mathrm{Bih})} u, I_{k}^{(\mathrm{Bih})} u\right)$ is minimal, we have $\rho\left(\left(I_{k}^{(\mathrm{Bih}, 2)}\right)^{*} I_{k}^{(\mathrm{Bih}, 2)}\right) \leq$ $\rho\left(\left(I_{k}^{(\mathrm{Bih}, 1)}\right)^{*} I_{k}^{(\mathrm{Bih}, 1)}\right)$.

Remark 3.1. In view of a practical implementation, we note that, in case of a multiplicative multi-grid method, if a prolongation $I_{k}^{(\mathrm{Bih})}$ is followed by a block GaussSeidel smoother for which the first block, that is assumed to be inverted exactly, corresponds to the degrees of freedom at the midpoints $m_{e}$ of $e \in E_{k} \backslash E_{k}^{(\text {new })}$, then the values $\partial_{\mathbf{n}_{e}}\left(I_{k}^{(\mathrm{Bih})} u\right)\left(m_{e}\right)$ for these $m_{e}$ are irrelevant. That is, when applying 


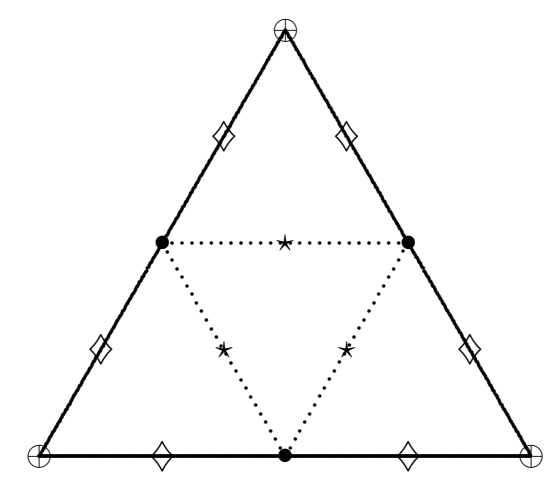

Figure 1. Degrees of freedom of $M_{k}$ : Vertices in $N_{k-1}(\oplus)$, or in $N_{k} \backslash N_{k-1}(\bullet)$; midpoints of $e \in E_{k}^{(\text {new })}(\star)$, or of $e \in E_{k} \backslash E_{k}^{\text {(new) }}$ $(\diamond)$

such a smoother, it does not matter whether $I_{k}^{(\mathrm{Bih}, 1)}$ or $I_{k}^{(\mathrm{Bih}, 2)}$ is used, and what is more, the values $\partial_{\mathbf{n}_{e}}\left(I_{k}^{(\mathrm{Bih})} u\right)\left(m_{e}\right)$ for these $m_{e}$ do not have to be computed. Obviously, an analogous comment applies to the restriction that is preceded by the adjoint smoother. The "trick" described here is well-known in the multi-grid literature in connection with multicolor Gauss-Seidel smoothing.

To be able to prove assumption (A), later on we will need the fact that, like $I_{k}^{(\mathrm{Bih}, 1)}$, the prolongation $I_{k}^{(\mathrm{Bih}, 2)}$ locally preserves second order polynomials: Let $\tilde{p} \in N_{k-1}$, and assume that $u \in M_{k-1}$ is equal to some second degree polynomial on the union $V$ of all $T \in \tau_{k-1}$ that contain $\tilde{p}$. Then $\partial_{\mathbf{n}_{e}}\left(I_{k}^{(\mathrm{Bih}, 2)} u\right)\left(m_{e}\right)=\partial_{\mathbf{n}_{e}} u\left(m_{e}\right)$ for all $e \in E_{k}^{(\text {new })}$, and, since $u$ is continuous on $V,\left(I_{k}^{(\operatorname{Bih}, 2)} u\right)(p)=u(p)$ for all $p \in N_{k}$ in the interior of $V$. Now let $U$ be the union of all triangles $T_{1}, \ldots, T_{q} \in \tau_{k}$ that contain $\tilde{p}$. Since the first order partial derivatives of $v \in \check{M}_{k}$ are continuous at $m_{e}$ for $e \in E_{k} \backslash E_{k}^{(\text {new })}$, and vanish at $m_{e}$ for $e \in E_{k}^{(\text {new })}$, integration by parts and an application of the midpoint quadrature rule shows that

$$
\sum_{\ell=1}^{q} \int_{T_{\ell}} \sum_{i, j} \frac{\partial^{2} u}{\partial x_{i} \partial x_{j}} \frac{\overline{\partial^{2} v}}{\partial x_{i} \partial x_{j}}=\sum_{\ell=1}^{q} \sum_{j} \int_{\partial T_{\ell}}\left(\nabla \frac{\partial u}{\partial x_{j}} \cdot \mathbf{n}\right) \frac{\overline{\partial v}}{\partial x_{j}}=0 .
$$

We conclude that $\left.\left(u-I_{k}^{(\mathrm{Bih}, 2)} u\right)\right|_{U}=0$, so that indeed $I_{k}^{(\mathrm{Bih}, 2)}$ locally preserves second order polynomials.

Since we were not able to derive useful theoretical upper bounds for the values of $\rho\left(\left(I_{j \leftarrow k}^{(\mathrm{Bih}, 2)}\right)^{*} I_{j \leftarrow k}^{(\mathrm{Bih}, 2)}\right)$, we give some numerical results for a model case.

Example 3.2. Let $\tau_{0}$ be the triangulation of $\Omega=[0,1]^{2}$ into two triangles, and for $k>0$, let $\tau_{k}$ be generated from $\tau_{k-1}$ by uniform dyadic refinement. Numerically computed values of $\rho\left(\left(I_{10 \leftarrow k}^{(\mathrm{Bih}, 2)}\right)^{*} I_{10 \leftarrow k}^{(\mathrm{Bih}, 2)}\right)$ and $\rho\left(\left(I_{10 \leftarrow k}^{(\mathrm{Bih}, 1)}\right)^{*} I_{10 \leftarrow k}^{(\mathrm{Bih}, 1)}\right)$ are given in Table 1 The results indicate that, in contrast to $\rho\left(\left(I_{j \leftarrow k}^{(\mathrm{Bih}, 1)}\right)^{*} I_{j \leftarrow k}^{(\mathrm{Bih}, 1)}\right)$, the values $\rho\left(\left(I_{j \leftarrow k}^{(\mathrm{Bih}, 2)}\right)^{*} I_{j \leftarrow k}^{(\mathrm{Bih}, 2)}\right)$ are uniformly bounded. The column $k=9$, however, shows that generally $\rho\left(\left(I_{k}^{(\mathrm{Bih}, 2)}\right)^{*} I_{k}^{(\mathrm{Bih}, 2)}\right)>2$, which means that also for $I_{k}^{(\mathrm{Bih}, 2)}$, when the number of smoothing steps is not large enough, the W-cycle might result in a preconditioned system that is indefinite. 
TABLE 1. $\rho\left(\left(I_{10 \leftarrow k}^{(\mathrm{Bih}, \mathrm{i})}\right)^{*} I_{10 \leftarrow k}^{(\mathrm{Bih}, \mathrm{i})}\right)$

\begin{tabular}{|c|c|c|c|c|c|c|c|c|c|c|}
\hline$k$ & 9 & 8 & 7 & 6 & 5 & 4 & 3 & 2 & 1 & 0 \\
\hline$i=2$ & 2.97 & 4.66 & 6.36 & 7.65 & 8.66 & 9.29 & 9.35 & 8.43 & 7.45 & 0.620 \\
\hline$i=1$ & 4.19 & $1.18 e 1$ & $3.05 e 1$ & $7.45 e 1$ & $1.76 e 2$ & $4.02 e 2$ & $8.64 e 2$ & $1.57 e 3$ & $1.93 e 3$ & $7.39 e 2$ \\
\hline
\end{tabular}

Remark 3.3. Instead of minimizing the energy norm over the degrees of freedom at the midpoints of $e \in E_{k} \backslash E_{k}^{(\text {new })}$, equally well we could have modified the standard prolongation $I_{k}^{(\mathrm{Bih}, 1)}$ by minimizing the energy with respect to the degrees of freedom at midpoints of $e \in E_{k}^{(\text {new })}$. Although obviously this also yields a prolongation with a smaller energy norm, in the model case of Example 3.2 the energy norm of the sufficiently many iterated prolongations turned out to be even larger than with the standard prolongation $I_{k}^{(\mathrm{Bih}, 1)}$.

For the following convergence analysis, we take \|\|$_{0, k}=\|\|_{L^{2}(\Omega)}$, which implies that $\rho_{k}$, defined in (2.8), satisfies $\rho_{k} \equiv 16^{k}$.

First we consider the additive multi-grid method. For the prolongation $I_{k}^{(\mathrm{Bih}, 1)}$, and with $P_{k-1}$ being the restriction to $M_{k}$ of the $a_{k}^{(\mathrm{Bih})}$ (, )-orthogonal projection from $M_{k}+M_{k-1}$ to $M_{k-1}$, in [Osw97] it was proved that the scalars $t_{j}$ from Theorem 2.12 are uniformly bounded. However, because of the generally exponential growth of $\rho\left(\left(I_{j \leftarrow k}^{(\mathrm{Bih}, 1)}\right)^{*} I_{j \leftarrow k}^{(\mathrm{Bih}, 1)}\right)$ as a function of $j \geq k$, from Remark 2.13 we learn that with this prolongation the condition numbers of the preconditioned system are exponentially growing as well.

If we replace $I_{k}^{(\mathrm{Bih}, 1)}$ by $I_{k}^{(\mathrm{Bih}, 2)}$ and use the same mappings $P_{k-1}$, then unfortunately the proof of $t_{j} \lesssim 1$ does not carry over. In view of [Osw97. Lemma 7], the problem is that, in contrast to $I_{k}^{(\mathrm{Bih}, 1)}$, the prolongation $I_{k}^{(\mathrm{Bih}, 2)}: M_{k-1} \rightarrow M_{k}$ cannot be extended to an $L^{2}(\Omega)$-bounded projector from $M_{k}+M_{k-1}$ onto $M_{k}$ (cf. also Remark 2.11). Yet, by definition of $P_{k}$, it follows that

$$
a_{k}^{(\mathrm{Bih})}\left(P_{k} u, P_{k} u\right)=a_{k+1}^{(\mathrm{Bih})}\left(P_{k} u, P_{k} u\right) \leq a_{k+1}^{(\mathrm{Bih})}(u, u) \quad\left(u \in M_{k+1}\right) .
$$

So if we can prove that

$$
\left\|\left(I-I_{k}^{(\mathrm{Bih}, 2)} P_{k-1}\right) u\right\|_{L^{2}(\Omega)}^{2} \lesssim 16^{-k} \sum_{T \in \tau_{k}}|u|_{H^{2}(T)}^{2} \quad\left(u \in M_{k}\right),
$$

then the suboptimal result $t_{j} \lesssim j$ is valid.

To show (3.9), we write

$$
I-I_{k}^{(\mathrm{Bih}, 2)} P_{k-1}=\left(I-I_{k}^{(\mathrm{Bih}, 2)}\right) P_{k-1}+\left(I-P_{k-1}\right) .
$$

In Osw97, it was proved that

$$
\left\|\left(I-P_{k-1}\right) u\right\|_{L^{2}(\Omega)}^{2} \lesssim 16^{-k} \sum_{T \in \tau_{k}}|u|_{H^{2}(T)}^{2} \quad\left(u \in M_{k}\right),
$$

which together with (3.8) and the following lemma shows (3.9) and thus $t_{j} \lesssim j$.

Lemma 3.4. With $I_{k}^{(\mathrm{Bih})}$ being either $I_{k}^{(\mathrm{Bih}, 1)}$ or $I_{k}^{(\mathrm{Bih}, 2)}$, we have

$$
\left\|\left(I-I_{k}^{(\mathrm{Bih})}\right) u\right\|_{L^{2}(\Omega)}^{2} \lesssim 16^{-k} \sum_{T \in \tau_{k-1}}|u|_{H^{2}(T)}^{2} \quad\left(u \in M_{k-1}\right) .
$$


Proof. By the exactness of the midpoint quadrature rule on first degree polynomials, in (3.6) we may replace $\partial_{\mathbf{n}_{e}} u\left(m_{e}\right)$ by $\frac{1}{|e|} \int_{e} \partial_{\mathbf{n}_{e}} u$, and in (3.4) we can make analogous replacements for $\partial_{\mathbf{n}_{e}}\left(\left.u\right|_{T_{i}}\right)\left(m_{e}\right)$. Although these modification thus do not change the definitions of the prolongations on $M_{k-1}$, in contrast to the original ones, the new definitions allow for canonical extensions of $I_{k}^{(\mathrm{Bih})}$ to mappings $\tilde{I}_{k}^{(\mathrm{Bih})}: M_{k-1}+$ $H_{0}^{2}(\Omega) \rightarrow M_{k}$. Since $\tilde{I}_{k}^{(\mathrm{Bih})}$ locally preserves first (even second) degree polynomials, the Bramble-Hilbert lemma and a homogeneity argument show that

$$
\left\|\left(I-\tilde{I}_{k}^{\mathrm{Bih})}\right) u\right\|_{L^{2}(\Omega)} \lesssim 4^{-k}\|u\|_{H^{2}(\Omega)} \quad\left(u \in H_{0}^{2}(\Omega)\right) .
$$

Let $\tilde{M}_{k-1} \subset H_{0}^{2}(\Omega)$ be the Hsieh-Clough-Tocher macro element space corresponding to $\tau_{k-1}$ (see, e.g., Cia78]). In [Bre99], a mapping $E_{k-1}: M_{k-1} \rightarrow \tilde{M}_{k-1}$ was constructed satisfying

$$
\left\|\left(I-E_{k-1}\right) u\right\|_{L^{2}(\Omega)}^{2} \lesssim 16^{-k} \sum_{T \in \tau_{k-1}}|u|_{H^{2}(T)}^{2} \quad\left(u \in M_{k-1}\right),
$$

and so, in particular,

$$
\left\|E_{k-1} u\right\|_{H^{2}(\Omega)}^{2} \lesssim \sum_{T \in \tau_{k-1}}|u|_{H^{2}(T)}^{2} \quad\left(u \in M_{k-1}\right) .
$$

A simple scaling argument shows that

$$
\left\|\tilde{I}_{k}^{(\mathrm{Bih})} u\right\|_{L^{2}(\Omega)} \lesssim\|u\|_{L^{2}(\Omega)} \quad\left(u \in M_{k-1}+\tilde{M}_{k-1}\right) .
$$

By writing $I-I_{k}^{(\mathrm{Bih})}=\left(I-\tilde{I}_{k}^{(\mathrm{Bih})}\right) E_{k-1}+\left(I-\tilde{I}_{k}^{(\mathrm{Bih})}\right)\left(I-E_{k-1}\right)$, the proof of the lemma follows from (3.10), (3.12), (3.13) and (3.11).

Assuming that indeed $\rho\left(\left(I_{j \leftarrow k}^{(\mathrm{Bih}, 2)}\right)^{*} I_{j \leftarrow k}^{(\mathrm{Bih}, 2)}\right) \lesssim 1$, from $t_{j} \lesssim j$ and Theorem 2.12 we conclude that with a Hermitian smoother that satisfies (2.23) the additive multigrid preconditioner $B_{j}^{(\text {add })}$ satisfies $\kappa\left(B_{j}^{(\text {add })} A_{j}\right) \lesssim j^{2}$.

We now turn to the verification of assumptions (A) and (B) for the multiplicative multi-grid method. It is well-known that the normalized bases corresponding to the degrees of freedom defining the Morley finite element space are uniformly $L^{2}(\Omega)$ stable. So Section 2.4 shows that (inexact) standard Gauss-Seidel and damped Jacobi smoothers satisfy assumption (B).

Using the local reproduction by both $I_{k}^{(\mathrm{Bih}, 1)}$ and $I_{k}^{(\mathrm{Bih}, 2)}$ of second order polynomials, a proof of assumption (A) with $\alpha=\frac{1}{2}$ can be deduced from [Bre99. Since with

$$
a^{(\mathrm{Bih})}(u, v):=\int_{\Omega} \sum_{i, j=1}^{2} \frac{\partial^{2} u}{\partial x_{i} \partial x_{j}} \frac{\overline{\partial^{2} v}}{\partial x_{i} \partial x_{j}}
$$

for $f \in H^{-2}(\Omega)$ the problem of finding $u \in H_{0}^{2}(\Omega)$ satisfying $a^{(\text {Bih })}(u, v)=f(v)$ $\left(v \in H_{0}^{2}(\Omega)\right)$ is not fully regular, i.e., $\|u\|_{H^{4}(\Omega)} \lesssim\|f\|_{L^{2}(\Omega)}$ is generally not valid, but instead only

$$
\|u\|_{H^{3}(\Omega)} \lesssim\|f\|_{H^{-1}(\Omega)}
$$

can be shown, we stress that assumption (A) with $\alpha>\frac{1}{2}$ cannot be expected. As a consequence, we may only conclude that the $\mathrm{W}$-cycle with a number of smoothing steps that is sufficiently large is a uniformly convergent iteration, and that the variable V-cycle yields preconditioned systems having uniformly bounded condition 
numbers. On the other hand, the mildly variable V-cycle does not necessarily yield uniformly bounded condition numbers, and, compared to an $\alpha=1$ case, less favourable results can be expected for the standard V-cycle. We will develop better V-cycle type methods in the next subsection.

3.3.2. An equivalent discretized Stokes problem. Let $\tau_{0}, \tau_{1}, \ldots$ be the sequence of triangulations as in Section 3.3.1 Let

$$
\mathbf{Z}_{k}=\left\{\mathbf{u} \in\left(V_{k}^{\left(P_{1}\right)}\right)^{2}: \operatorname{div}_{k} \mathbf{u}=0\right\}
$$

where $V_{k}^{\left(P_{1}\right)}$ is the nonconforming $P_{1}$ space from Section [3.1, and $\left.\left(\operatorname{div}_{k} \mathbf{u}\right)\right|_{T}:=$ $\left.\operatorname{div} \mathbf{u}\right|_{T}\left(T \in \tau_{k}\right)$.

With $\left.\left(\operatorname{curl}_{k} v\right)\right|_{T}:=\left.\operatorname{curl} v\right|_{T}\left(T \in \tau_{k}\right)$, in [FM90] it was proved that $\operatorname{curl}_{k}$ : $M_{k} \rightarrow \mathbf{Z}_{k}$ is a bijection, and moreover that

$$
a_{k}^{(\mathrm{Bih})}(u, v)=a_{k}^{(\mathrm{St})}\left(\operatorname{curl}_{k} u, \operatorname{curl}_{k} v\right)
$$

where

$$
a_{k}^{(\mathrm{St})}(\mathbf{u}, \mathbf{v}):=\sum_{T \in \tau_{k}} \int_{T} \sum_{i=1}^{2} \nabla \mathbf{u}_{i} \cdot \nabla \mathbf{v}_{i} .
$$

So when $g=\mathbf{f} \circ \mathbf{c u r l}_{j}$, the problems of solving

$$
a_{j}^{(\mathrm{Bih})}(u, v)=g(v) \quad\left(v \in M_{j}\right)
$$

and

$$
a_{j}^{(\mathrm{St})}(\mathbf{u}, \mathbf{v})=\mathbf{f}(\mathbf{v}) \quad\left(\mathbf{v} \in \mathbf{Z}_{j}\right)
$$

are equivalent, in the sense that $\mathbf{u}=\operatorname{curl}_{j} u$.

Remark 3.5. The equation (3.17) can be identified as characterizing the velocity components of a discretized Stokes problem. In $\underline{\mathrm{Ste} 00}$, an efficient and stable postprocessing procedure is presented for finding an approximation for the pressure component assuming that some approximations of the velocity components are available.

A consequence of the equivalence of (3.16) and (3.17) is that if on all levels we relate smoothers and prolongations for both problems according to

$$
c_{k}^{(\mathrm{Bih})}(u, v)=c_{k}^{(\mathrm{St})}\left(\operatorname{curl}_{k} u, \operatorname{curl}_{k} v\right)
$$

and

$$
\operatorname{curl}_{k} I_{k}^{(\mathrm{Bih})}=I_{k}^{(\mathrm{St})} \operatorname{curl}_{k-1},
$$

then the resulting multi-grid methods are equivalent. Moreover, if we equip $\mathbf{Z}_{k}$ with a basis generated by applying $\operatorname{curl}_{k}$ to all basis functions of $M_{k}$, then from Section 2.2 it appears that for both multi-grid methods the matrix representations $\mathbf{A}_{k}, \mathbf{p}_{k}, \mathbf{p}_{k}^{T}, \mathbf{C}_{k}^{-1}$ and $\mathbf{C}_{k}^{-H}$ of all individual components are equal, and also that the vector representations of the right-hand sides and the solutions are equal.

This equivalence of both multi-grid methods was used earlier in the literature, e.g., in [Bre90, in the sense that the discretized biharmonic was used to analyze the behaviour of the multi-grid method applied to the discretized Stokes problem. Here we will follow the opposite approach. 
In our general nonconforming multi-grid framework, let $V_{k}=\mathbf{Z}_{k}$ and $a_{k}=a_{k}^{(\mathrm{St})}$. With \|\|$_{0, k}:=\|\|_{L^{2}(\Omega)^{2}}$, we find that $\rho_{k} \overline{\bar{\sim}} 4^{k}$. Since an analysis using the Stokes formulation of the additive method does not yield new insights, we concentrate on the multiplicative multi-grid method. In the setting of Section 2.5, we take

$$
\mathcal{H}^{0}=L^{2}(\Omega)^{2}, \quad \mathcal{H}^{1}=\left\{\mathbf{u} \in H_{0}^{1}(\Omega)^{2}: \operatorname{div} \mathbf{u}=0\right\}, \quad \mathcal{H}^{2}=H^{2}(\Omega)^{2} \cap \mathcal{H}^{1}
$$

and $a=a^{(\mathrm{St})}$ defined by

$$
a^{(\mathrm{St})}(\mathbf{u}, \mathbf{v})=\int_{\Omega} \sum_{i=1}^{2} \nabla \mathbf{u}_{i} \cdot \nabla \mathbf{v}_{i}
$$

With these definitions, conditions arc of Theorem 2.10 are satisfied. In fact, using the continuous equivalent of (3.15), condition ("full" regularity) can be shown to be equivalent to (3.14). For the verification of $\mathrm{b}$ and $\mathrm{c}$ one may consult [Ste99. $\S 6.3]$, where they correspond to conditions (I) and $(\mathrm{G})$, respectively.

Because curl : $H_{0}^{2}(\Omega) \cap H^{3}(\Omega) \rightarrow \mathcal{H}^{2}$ is a homeomorphism (see, e.g., [GR86]), the conditions $\mathrm{d}] \mathrm{f}$ of Theorem 2.10 involving $I_{k}^{(\mathrm{St})}: \mathbf{Z}_{k-1} \rightarrow \mathbf{Z}_{k}$ and some suitable $\Pi_{k}^{(\mathrm{St})}: \mathcal{H}^{2} \rightarrow \mathbf{Z}_{k}$ can be rewritten as

$$
\sum_{T \in \tau_{k}}\left|\left(I-\Pi_{k}^{(\mathrm{Bih})}\right) u\right|_{H^{1}(T)}^{2} \lesssim 16^{-k}\|u\|_{H^{3}(\Omega)}^{2} \quad\left(u \in H_{0}^{2}(\Omega) \cap H^{3}(\Omega)\right),
$$

$$
\sum_{T \in \tau_{k}}\left|\left(\Pi_{k}^{(\mathrm{Bih})}-I_{k}^{(\mathrm{Bih})} \Pi_{k-1}^{(\mathrm{Bih})}\right) u\right|_{H^{1}(T)}^{2} \lesssim 16^{-k}\|u\|_{H^{3}(\Omega)}^{2} \quad\left(u \in H_{0}^{2}(\Omega) \cap H^{3}(\Omega)\right),
$$

and

$$
\sum_{T \in \tau_{k}}\left|I_{k}^{(\mathrm{Bih})} u\right|_{H^{1}(T)}^{2} \lesssim \sum_{T \in \tau_{k-1}}|u|_{H^{1}(T)}^{2} \quad\left(u \in M_{k-1}\right)
$$

where $I_{k}^{(\mathrm{Bih})}, I_{k}^{(\mathrm{St})}$ and $\Pi_{k}^{(\mathrm{Bih})}, \Pi_{k}^{(\mathrm{St})}$ are related according to $(3.18)$ and $\mathbf{c u r l}_{k} \Pi_{k}^{(\mathrm{Bih})}$ $=\Pi_{k}^{(\mathrm{St})}$ curl, respectively.

Let $I_{k}^{(\mathrm{Bih})}$ be either $I_{k}^{(\mathrm{Bih}, 1)}$ or $I_{k}^{(\mathrm{Bih}, 2)}$, and let $\Pi_{k}^{(\mathrm{Bih})}$ be defined by $\left(\Pi_{k}^{(\mathrm{Bih})} u\right)(p)=$ $u(p)\left(p \in N_{k}\right)$ and $\partial_{\mathbf{n}_{e}}\left(\Pi_{k}^{(\mathrm{Bih})} u\right)\left(m_{e}\right)=\partial_{\mathbf{n}_{e}} u\left(m_{e}\right)\left(e \in E_{k}\right)$. Then, using the local reproduction by $\Pi_{k}^{(\mathrm{Bih})}$ and $I_{k}^{(\mathrm{Bih})}$ of second order polynomials, (3.20) and (3.21) follow from the Bramble-Hilbert lemma and a homogeneity argument. The inverse inequalities $|\cdot|_{H^{2}(T)} \lesssim 2^{k}|\cdot|_{H^{1}(T)}$ and $|\cdot|_{H^{1}(T)} \lesssim 2^{k}\|\cdot\|_{L^{2}(T)}$ on $P_{2}(T), T \in \tau_{k}$, together with Lemma 3.4 show (3.21). Theorem 2.10 shows that within this Stokes framework assumption (A) with $\alpha=1$ is valid.

Now we turn to the verification of assumption $(\mathrm{B})$. Applying $\mathbf{c u r l}_{k}$ to the canonical basis of $M_{k}$ that corresponds to the degrees of freedom defining this space, we obtain a basis for $\mathbf{Z}_{k}$ that we denote by

$$
\left\{\mathbf{v}_{k, e}: e \in E_{k}\right\} \cup\left\{\mathbf{w}_{k, p}: p \in N_{k}\right\} \text {. }
$$

One may verify that

$$
\mathbf{v}_{k, e}=\eta_{k, e} \mathbf{t}_{e}
$$



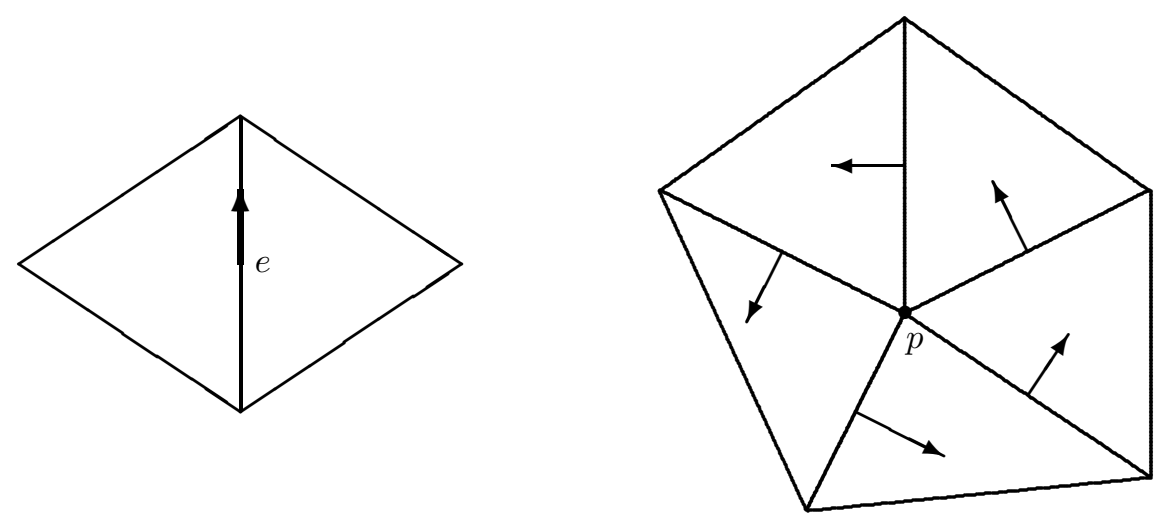

Figure 2. Basis functions $\mathbf{v}_{k, e}$ and $\mathbf{w}_{k, p}$ of the space $\mathbf{Z}_{k}$

where $\mathbf{t}_{e}=\left[\left(\mathbf{n}_{e}\right)_{2}-\left(\mathbf{n}_{e}\right)_{1}\right]^{T}$ is a unit vector tangential to $e$ and $\eta_{k, e}$ is the canonical basis function of $V_{k}^{\left(P_{1}\right)}$ corresponding to $e$ defined in (3.1), and furthermore that

$$
\mathbf{w}_{k, p}=\sum_{i=1}^{\ell}\left|e_{i}\right|^{-1} \eta_{k, e_{i}} \mathbf{n}_{e_{i}, p},
$$

where $e_{1}, \ldots, e_{\ell} \in E_{k}$ are all edges that contain $p$, and $\mathbf{n}_{e_{i}, p}$ is the unit vector normal to $e_{i}$ pointing in the counterclockwise direction with respect to $p$, see Figure 2

Using the fact that $\left\{\eta_{k, e}: e \in E_{k}\right\}$ is an $L^{2}(\Omega)$-orthogonal basis for $V_{k}^{\left(P_{1}\right)}$, for vectors $\mathbf{c}=\left(\mathbf{c}_{e}\right)_{e \in E_{k}}$ and $\mathbf{d}=\left(\mathbf{d}_{p}\right)_{p \in N_{k}}$, we infer that

$$
\left\|\sum_{e \in E_{k}} \mathbf{c}_{e} \mathbf{v}_{k, e}+\sum_{p \in N_{k}} \mathbf{d}_{p} \mathbf{w}_{k, p}\right\|_{L^{2}(\Omega)^{2}}^{2}=\sum_{e \in E_{k}}\left|\mathbf{c}_{e}\right|^{2}\left\|\eta_{k, e}\right\|_{L^{2}(\Omega)}^{2}+\left\|\sum_{p \in N_{k}} \mathbf{d}_{p} \mathbf{w}_{k, p}\right\|_{L^{2}(\Omega)^{2}}^{2}
$$

and

$$
\left\|\sum_{p \in N_{k}} \mathbf{d}_{p} \mathbf{w}_{k, p}\right\|_{L^{2}(\Omega)^{2}}^{2}=\sum_{e \in E_{k}}\left|\mathbf{d}_{p_{e}}-\mathbf{d}_{\tilde{p}_{e}}\right|^{2}|e|^{-2}\left\|\eta_{k, e}\right\|_{L^{2}(\Omega)}^{2},
$$

where $p_{e}, \tilde{p}_{e} \in \bar{N}_{k}$ denote both vertices on $e \in E_{k}$, and $\mathbf{d}_{p}:=0$ when $p \in \bar{N}_{k} \backslash N_{k}$. From (3.24) we conclude that the normalized bases (3.22) are not uniformly $L^{2}(\Omega)^{2}$ stable, and so that general Gauss-Seidel or damped Jacobi smoothers do not necessarily satisfy assumption (B).

Now, let $\mathbf{Z}_{k}^{(0)}=\operatorname{span}\left\{\mathbf{w}_{k, p}: p \in N_{k}\right\}$, and let $\mathbf{Z}_{k}^{(i)}=\operatorname{span}\left\{\mathbf{v}_{k, e}: e \in E_{k}^{(i)}\right\}$ for $1 \leq i \leq m$, where $\bigcup_{i=1}^{m} E_{k}^{(i)}$ is some partition of $E_{k}$ into disjoint subsets. Then by definition of $\rho_{k}$ and (3.23), we have

$$
\sum_{i=0}^{m} a_{k}^{(\mathrm{St})}\left(\mathbf{u}^{(i)}, \mathbf{u}^{(i)}\right) \leq \rho_{k} \sum_{i=0}^{m}\left\|\mathbf{u}^{(i)}\right\|_{L^{2}(\Omega)^{2}}^{2}=\rho_{k}\left\|\sum_{i=0}^{m} \mathbf{u}^{(i)}\right\|_{L^{2}(\Omega)^{2}}^{2} \quad\left(\mathbf{u}^{(i)} \in \mathbf{Z}_{k}^{(i)}\right) .
$$

That is, if, with respect to the decomposition $\mathbf{Z}_{k}=\bigoplus_{i=0}^{m} \mathbf{Z}_{k}^{(i)}, \mathbf{D}_{k}$ is the block diagonal part of the stiffness matrix $\mathbf{A}_{k}$ corresponding to (3.22), then condition ఏ of Proposition 2.4 is satisfied. So, if in addition for $i>0$ the $\mathbf{Z}_{k}^{(i)}$ are selected such that, possibly after reordering, the decomposition $\mathbf{Z}_{k}=\bigoplus_{i=0}^{m} \mathbf{Z}_{k}^{(i)}$ satisfies 
C of Proposition 2.4 as well, then these resulting block Gauss-Seidel and damped block Jacobi smoothers do satisfy (B).

Since neither of the spaces $\mathbf{Z}_{k}^{(i)}$ contain smooth vector fields, we infer that

$$
a^{(\mathrm{St})}\left(\mathbf{u}^{(i)}, \mathbf{u}^{(i)}\right) \equiv \rho_{k}\left\|\mathbf{u}^{(i)}\right\|_{L^{2}(\Omega)^{2}}^{2} \quad\left(\mathbf{u}^{(i)} \in \mathbf{Z}_{k}^{(i)}, 0 \leq i \leq m\right) .
$$

So, in particular, 3.25) for $i=0$ combined with (3.24) shows that a further decomposition of $\mathbf{Z}_{k}^{(0)}$ into subspaces each of them spanned by some uniformly bounded number of $\mathbf{w}_{k, p}$ 's will generally not give rise to (block) Gauss-Seidel or damped (block) Jacobi smoothers that satisfy $(B)$, because condition a of Proposition 2.4 will be violated.

At the same time, (3.25) for $i=0$ combined with (3.24) shows that "exact" block Gauss-Seidel or damped block Jacobi smoothers corresponding to $\mathbf{Z}_{k}=\bigoplus_{i=0}^{m} \mathbf{Z}_{k}^{(i)}$ are not feasible, since the diagonal block of $\mathbf{D}_{k}$ corresponding to $\mathbf{Z}_{k}^{(0)}$ cannot be inverted in $\mathcal{O}\left(\operatorname{dim} \mathbf{Z}_{k}\right)$ operations. However, from Propositions 2.4 and 2.7 we learn that in order to satisfy (B), it is sufficient to invert the diagonal blocks approximately, at least when the approximate inverses define iterations that converge uniformly in the corresponding "energy" norms.

Considering the diagonal block corresponding to $\mathbf{Z}_{k}^{(0)}$, one easily verifies that

$$
\sum_{e \in E_{k}}\left|\mathbf{d}_{p_{e}}-\mathbf{d}_{\tilde{p}_{e}}\right|^{2}|e|^{-2}\left\|\eta_{k, e}\right\|_{L^{2}(\Omega)}^{2} \equiv \int_{\Omega}\left|\nabla d^{I}\right|^{2} d x
$$

where $d^{I}$ is the function in the conforming $P_{1}$ finite element space $C(\bar{\Omega}) \cap H_{0}^{1}(\Omega) \cap$ $\prod_{T \in \tau_{k}} P_{1}(T)$ defined by $d^{I}(p)=\mathbf{d}_{p}\left(p \in N_{k}\right)$. Optimal conforming multi-grid preconditioners that take only $\mathcal{O}\left(\# N_{k}\right)$ operations are available for the right-hand side of (3.26). So properly scaled, these preconditioners satisfy the assumptions to be used as inexact solvers for the diagonal block of $\mathbf{D}_{k}$ corresponding to $\mathbf{Z}_{k}^{(0)}$.

If not already invertible in $\mathcal{O}\left(\operatorname{dim} \mathbf{Z}_{k}\right)$ operations, (3.25) for $i>0$ and (3.23) show that the other diagonal blocks of $\mathbf{D}_{k}$ are uniformly well-conditioned, so that also for these blocks suitable approximate inverses are available. From Proposition 2.4 or [2.7, we conclude that the above introduced "inexact" block Gauss-Seidel or damped block Jacobi smoothers satisfy assumption $(\mathrm{B})$, and that they can be performed in $\mathcal{O}\left(\operatorname{dim} \mathbf{Z}_{k}\right)$ operations.

Since, in this Stokes framework, (A) with $\alpha=1$ and, with the above smoothers, (B) are valid, compared to the discretized biharmonic formulation from Section 3.3.1, we obtain the following new results: The mildly variable V-cycle yields preconditioned systems that have uniformly bounded condition numbers. Furthermore, assuming that $\rho\left(\left(\tilde{I}_{j \leftarrow k}^{(\mathrm{St}, 2)}\right)^{*} \tilde{I}_{j \leftarrow k}^{(\mathrm{St}, 2)}\right) \lesssim 1$, the standard V-cycle using the prolongation $I_{k}^{(\mathrm{St}, 2)}$ yields a preconditioner that is at least suboptimal. This condition on the "iterated prolongations alternated with smoothers" likely follows from $\rho\left(\left(I_{j \leftarrow k}^{(\mathrm{St}, 2)}\right)^{*} I_{j \leftarrow k}^{(\mathrm{St}, 2)}\right)=\rho\left(\left(I_{j \leftarrow k}^{(\mathrm{Bih}, 2)}\right)^{*} I_{j \leftarrow k}^{(\mathrm{Bih}, 2)}\right) \lesssim 1$ (for completeness, here ()$^{*}$ refers to energy scalar products in the Stokes and biharmonic framework, respectively). Numerical evidence for the latter result was found in the model case of Example 3.2 . 
3.3.3. Practical algorithms and numerical results. We apply the multiplicative standard V-cycle to the discretized biharmonic problem (3.16), or equivalently, the discretized Stokes problem (3.17), taking $m(k) \equiv m=1$, i.e., one post-smoothing step and one pre-smoothing step with the "adjoint" smoother.

We use either the standard prolongation

- $I_{k}^{(\mathrm{Bih}, 1)}\left(I_{k}^{(\mathrm{St}, 1)}\right)$,

or the new prolongation

- $I_{k}^{(\mathrm{Bih}, 2)}\left(I_{k}^{(\mathrm{St}, 2)}\right)$.

Equipping $M_{k}$ or $\mathbf{Z}_{k}$ with the standard basis (3.22), we use "inexact" block Gauss-Seidel smoothing with respect to the following ordered subdivision of the degrees of freedom:

1. midpoints of $e \in E_{k} \backslash E_{k}^{\text {(new) }}$,

2. vertices,

3. midpoints of $e \in E_{k}^{(\text {new })}$,

and with reversed ordering in the post-smoothing step.

Assuming uniform dyadic refinements, note that with respect to this partitioning the first and last diagonal block of the stiffness matrix $\mathbf{A}_{k}$ are block diagonal matrices with blocks of small and uniformly bounded sizes. We invert the third diagonal block exactly, and the first one approximately, by applying one damped point Jacobi iteration with $\omega=1$ (cf. Remark 2.9).

We consider two options to approximate the inverse of the second diagonal block. We apply either

- one damped point Jacobi iteration with $\omega=1$

or

- a properly scaled multi-grid iteration for a discretized Laplacian on the corresponding conforming $P_{1}$ finite element space.

As demonstrated in $\$ \$ 3.3 .1$ and 3.3.2, the smoother corresponding to the first option satisfies $(\mathbb{B})$ in the biharmonic framework but not in the Stokes framework, whereas the smoother corresponding to the second option satisfies $(\mathrm{B})$ also in the Stokes framework. The first smoother can be expected to give qualitatively similar results as any arbitrary smoother, and we will refer to this smoother as the "standard smoother". The second smoother will be referred to as the "new smoother".

As noted in Remark [3.1, the combination of either smoother with $I_{k}^{(\mathrm{Bih}, 2)}$ can be implemented efficiently by replacing both the computation of the normal derivatives of the prolongated function at the midpoints of $e \in E_{k} \backslash E_{k}^{(\text {new })}$ and the application of the approximate inverse of the first diagonal block, by the application of the exact inverse of this block.

We have performed numerical tests in the model case of $\tau_{0}$ being the subdivision of $\Omega=[0,1]^{2}$ into two triangles, and for $k>0, \tau_{k}$ being generated from $\tau_{k-1}$ by uniform dyadic refinement. In this case, the second diagonal block of $\mathbf{A}_{k}$ is just a multiple of the discretized Laplacian on the corresponding conforming $P_{1}$ finite element space. For the new smoother, as "inner" multi-grid method we applied one standard V-cycle with one block Gauss-Seidel iteration with respect to a "redblack" ordering of the unknowns as a pre-smoother, and one "adjoint" iteration as the post-smoother. Note that Propositions 2.4 and 2.7]also allow for "unsymmetric" inner multi-grid methods, but we did not test this possibility. 
TABLE 2. Operation count per degree of freedom

\begin{tabular}{|c|c|c|}
\hline & $I_{k}^{(\mathrm{Bih}, 1)}$ & $I_{k}^{(\mathrm{Bih}, 2)}$ \\
\hline standard smoother & 56 & 45 \\
\hline new smoother & 70 & 57 \\
\hline
\end{tabular}

For all four combinations of $I_{k}^{(\mathrm{Bih}, 1)}$ or $I_{k}^{(\mathrm{Bih}, 2)}$, and standard or new smoother, we counted the number of arithmetic operations per degree of freedom necessary to perform one multi-grid iteration, where we let the number of levels tend to infinity. The results given in Table 2 show that implementing together $I_{k}^{(\operatorname{Bih}, 2)}$ and the new smoother does not increase the costs compared to $I_{k}^{(\mathrm{Bih}, 1)}$ and the standard smoother. Note that, on a regular mesh, the number of vertices is only $\frac{1}{4}$ of the total number of degrees of freedom of a Morley finite element space, which explains why the new smoother needs relatively few additional operations.

Although for nonregular meshes the operation counts will be somewhat larger, the ratios will basically be the same.

Numerically computed condition numbers of the stiffness matrix $\mathbf{A}_{j}$ preconditioned with the standard V-cycle with each of the four smoother-prolongation combinations are presented in Figure 3. The results show that the condition numbers for the standard method increase almost exponentially with the number of levels. Both the new smoother and the new prolongation result in smaller condition numbers. With both improvements implemented, the condition numbers are "small" and they even appear to be uniformly bounded.

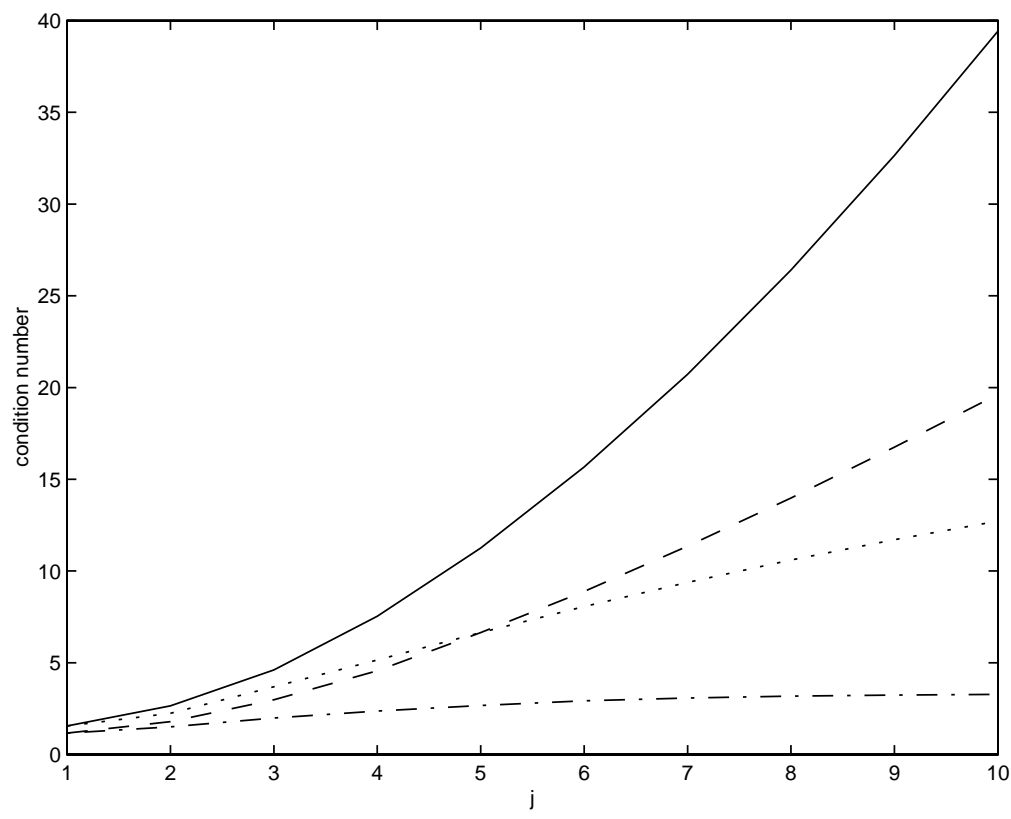

FIGURE 3. Standard smoother, standard prolongation (-); new smoother, standard prolongation $(--)$; standard smoother, new prolongation $(\cdots)$; new smoother, new prolongation $(-\cdot)$ 


\section{REFERENCES}

[BDH99] D. Braess, M. Dryja, and W. Hackbusch. A multigrid method for nonconforming FEdiscretisations with applications to non-matching grids. Computing, 63(1):1-25, 1999. MR 2000h:65048

[BP92] J.H. Bramble and J.E. Pasciak. The analysis of smoothers for multigrid algorithms. Math. Comp., 58:467-488, 1992. MR 92f:65146

[BPX91] J.H. Bramble, J.E. Pasciak, and J. Xu. The analysis of multigrid algorithms with nonnested spaces or inherited quadratic forms. Math. Comp., 56(193):1-34, 1991. MR 91h:65159

[Bre89] S.C. Brenner. An optimal-order nonconforming multigrid method for the biharmonic equation. SIAM J. Numer. Anal, 26(5):1124-1138, 1989. MR 89f:65119

[Bre90] S.C. Brenner. A nonconforming multigrid method for the stationary Stokes equations. Math. Comp., 55(192):411-437, 1990. MR 91d:65167

[Bre99] S.C. Brenner. Convergence of nonconforming multigrid methods without full elliptic regularity. Math. Comp., 68(225):25-53, 1999. MR 99c:65229

[BS94] S.C. Brenner and L.R. Scott. The Mathematical Theory of Finite Element Methods. Springer-Verlag, New York, 1994. MR 95f:65001

[Che97] Z. Chen. The analysis of intergrid transfer operators and multigrid methods for nonconforming finite elements. ETNA, 5:78-96, 1997. MR 99c:65230

[Che99] Z. Chen. On the convergence of Galerkin-multigrid methods for nonconforming finite elements. East-West J. Numer. Math., 7(2):79-104, 1999. MR 2000c:65116

[Cia78] P.G. Ciarlet. The finite element method for elliptic problems. North-Holland, Amsterdam, 1978. MR 58:25001

[CO98] Z. Chen and P. Oswald. Multigrid and multilevel methods for nonconforming rotated Q1 elements. Math. Comp., 67:667-693, 1998. MR 98g:65118

[FM90] R.S. Falk and M.E. Morley. Equivalence of finite element methods for problems in elasticity. SIAM J. Numer. Anal., 27(6):1486-1505, 1990. MR 91i:65117

[GR86] V. Girault and P.A. Raviart. Finite element methods for Navier-Stokes equations, Theory and Algorithms. Springer-Verlag, Berlin, 1986. MR 88b:65129

[Hac85] W. Hackbusch. Multi-Grid Methods and Applications. Springer-Verlag, Berlin, 1985.

[MMB87] J. Mandel, S. McCormick, and R. Bank. Variational multigrid theory. In S.F. McCormick, editor, Multigrid Methods, chapter 5. SIAM, Philadelphia, Pennsylvania, 1987, pp. 131-177. MR 89m:65004

[Osw92] P. Oswald. On a hierarchical basis multilevel method with nonconforming P1 elements. Numer. Math., 62:189-212, 1992. MR 93b:65059

[Osw97] P. Oswald. Intergrid transfer operators and multilevel preconditioners for nonconforming discretizations. Appl. Numer. Math, 23, 1997, 139-158. MR 98g:65110

[Ste99] R.P. Stevenson. Nonconforming finite elements and the cascadic multi-grid method. Technical Report 1120, University of Utrecht, November 1999. Revised version, January 2001, to appear in Numer. Math.

[Ste00] R.P. Stevenson. A direct solver for the gradient equation. Technical Report 1163, University of Utrecht, October 2000. to appear in Math. Comp.

Department of Mathematics, Utrecht University, P.O. Box 80.010, NL-3508 TA UtRecht, The NetherLANDS

E-mail address: stevenso@math.uu.nl 